\title{
Logarithmic black hole entropy corrections and holographic Rényi entropy
}

\author{
Subhash Mahapatra ${ }^{1,2, a}$ \\ ${ }^{1}$ The Institute of Mathematical Sciences, Chennai 600113, India \\ 2 Department of Physics, KU Leuven Campus Kortrijk - KULAK, Etienne Sabbelaan 53 bus 7800, 8500 Kortrijk, Belgium
}

Received: 11 October 2017 / Accepted: 29 December 2017 / Published online: 12 January 2018

(C) The Author(s) 2018. This article is an open access publication

\begin{abstract}
The entanglement and Rényi entropies for spherical entangling surfaces in CFTs with gravity duals can be explicitly calculated by mapping these entropies first to the thermal entropy on hyperbolic space and then, using the AdS/CFT correspondence, to the Wald entropy of topological black holes. Here we extend this idea by taking into account corrections to the Wald entropy. Using the method based on horizon symmetries and the asymptotic Cardy formula, we calculate corrections to the Wald entropy and find that these corrections are proportional to the logarithm of the area of the horizon. With the corrected expression for the entropy of the black hole, we then find corrections to the Rényi entropies. We calculate these corrections for both Einstein and Gauss-Bonnet gravity duals. Corrections with logarithmic dependence on the area of the entangling surface naturally occur at the order $G_{D}^{0}$. The entropic $c$-function and the inequalities of the Rényi entropy are also satisfied even with the correction terms.
\end{abstract}

\section{Introduction}

Quantum entanglement is one of the most remarkable properties of quantum systems; it is essential to most quantuminformation applications. The quantification and characterization of entanglement is an important problem in quantuminformation science and a number of measures have been suggested for its definition in the literature [1]. Two such measures are entanglement and Rényi entropies. Formally, given a subsystem $v$ and its complement $\bar{v}$, the entanglement entropy of the subsystem $v$ is defined by the von Neumann entropy of its reduced density matrix $\rho_{v}$,

$S_{\mathrm{EE}}=-\operatorname{Tr}\left[\rho_{v} \ln \rho_{v}\right]$,

\footnotetext{
a e-mails: subhash.mahapatra@ kuleuven.be; subhashmahapatra@gmail.com
}

where $\rho_{v}$ is obtained by tracing out the degrees of freedom of the subsystem $\bar{v}$ from the full density matrix. Similarly, the Rényi entropy is defined by a one-parameter generalization of the von Neumann entropy,

$S_{q}=\frac{1}{1-q} \ln \operatorname{Tr}\left[\rho_{v}^{q}\right]$,

where $q$ is a positive real number. Given the above definition, one finds that $\lim _{q \rightarrow 1} S_{q}=S_{\mathrm{EE}}$, i.e., the entanglement entropy is recovered from the Rényi entropy by taking the limit $q \rightarrow 1$.

The properties of entanglement and Rényi entropies have been the subject of intense investigations in the last two decades and form the basis for many applications ranging from condensed matter physics to quantum gravity. The standard way of calculating these entropies in quantum field theories is the replica method [2]. In this method, one maps the trace of the $q$ th power of the density matrix $\rho_{v}$ to the partition function on a singular $q$-folded Riemann surface. Geometrically, this $q$-fold space is a flat cone with angle deficit $2 \pi(1-q)$ at the entangling surface, and the Euclidean path integral over fields defined on this $q$-fold space can be explicitly computed for a few simple cases. However, for generic quantum field theories the computation of the partition function on the singular surfaces is rather difficult, which limits the usefulness of the replica method.

On the other hand, recent developments in the AdS/CFT correspondence [3] have suggested an elegant and geometric way of computing entanglement entropy in conformal field theories (CFTs) which have gravity duals. In the seminal work of Ryu and Takayanagi (RT) [4,5], the entanglement entropy of a $d$-dimensional boundary CFT was conjectured to be given by the area of a minimal surface in the bulk AdS space as

$S_{\mathrm{EE}}=\frac{\operatorname{Area}\left(\gamma_{v}\right)}{4 G_{D}}$, 
where $\gamma_{v}$ is the $(d-1)$-dimensional minimal-area hypersurface, which extends into the bulk spacetime and shares the same boundary $\partial v$ of the subsystem $v$. This proposal has been extensively tested for a variety of systems and by now there is a good amount of evidence to support this conjecture [6]. Indeed, further indications for the correctness of this conjecture was provided in [7], where the generalized replica method was applied in the bulk AdS space to prove the RT conjecture.

An alternative approach to calculating the holographic entanglement entropy, which works only for spherical entangling surfaces, was proposed in [8]. In [8], it was observed that the reduced density matrix for a spherical entangling region in flat space can be conformally mapped to the thermal density matrix on the hyperbolic space; or equivalently the original entanglement entropy can be mapped to thermal entropy on the hyperbolic space [9]. Then, using the AdS/CFT correspondence, the latter thermal entropy can be again mapped on the dual gravity side to black hole entropy of certain topological black holes with hyperbolic horizons. This observation therefore also provided an alternative derivation of the RT conjecture for a spherical entangling surface in $d$-dimensional CFT. Moreover, in [10], it was observed that the idea of [8] can be further extended to the study of the Rényi entropy for a spherical entangling region. For the record, let us also mention that a holographic variant for the Rényi entropy, which works for any entangling surface, has recently been put forth in [11]. See also $[12,13]$, for a discussion of the holographic Rényi entropy in two-dimensional boundary CFTs.

The procedure of mapping entanglement and Rényi entropies of the boundary CFT to the entropy of the black hole have many advantages. In particular, this mapping can easily be generalized to calculate Rényi entropy of higherdimensional CFTs. Moreover, this mapping can also be generalized to calculate Rényi entropy of boundary theories which are dual to higher derivative gravity theories.

An essential point which comes in the derivation of $[8,10]$ is the notion of black hole entropy, which can be computed using the standard Wald formula. Using the Wald entropy, the above procedure indeed reproduces independent known results of Rényi entropy. However, an important point, which we want to stress here and that will play a significant role in this paper, is that the Wald entropy only gives the leading order contribution to the entropy of the black hole. There are additional subleading quantum corrections to the usual Wald entropy, which are generally proportional to the logarithm of the area of the horizon. Indeed, recent activities in most quantum gravity models, including string theory and loop quantum gravity (LQG) or methods based on diffeomorphism symmetry arguments, have predicted logarithmic corrections to the entropy of the black hole, with a general expression like
$S=S_{\text {Wald }}+\mathbf{C} \ln S_{\text {Wald }}+\cdots$.

The coefficient $\mathbf{C}$ has been calculated for a variety of cases. For example, for the Schwarzschild black hole the coefficient $\mathbf{C}$ was found to be equal to $-3 / 2$ in LQG [14]. There, the horizon was treated as a boundary spacetime which was quantized within the "quantum geometry" program. Similarly in string theory, using the Euclidean gravity approach, logarithmic corrections to the entropy of extremal and nonextremal asymptotically flat black holes have been computed in [15-18], also see [19-22]. There, the logarithmic correction arises at the one-loop level from the massless fields of the theory and the coefficient $\mathbf{C}$ was found to be dependent on the total number of spacetime dimensions as well as the number of massless fields. A simple comparison showed disagreement on the value of $\mathbf{C}$ in these two quantum gravity models. ${ }^{1}$

The black hole entropy and its logarithmic correction can also be calculated using an approach based on the argument of diffeomorphism symmetry on the horizon. This approach, using the work of [28], was initiated in [29] and later emphasized in [30-32] to calculate black hole entropy. In this approach one first identifies a set of vector fields (based on some physical consideration) and then construct an algebra for the Fourier modes of the charges (corresponding to an appropriate diffeomorphism symmetry) from these vector fields. Using this algebra, which usually has a twodimensional Virasoro algebra like structure, one then extracts the central charge and the zero mode from it and then finally uses the Cardy formula [34] for the asymptotic density of states to compute the entropy of the black hole. It has been shown in [30-32] that this approach does indeed correctly reproduce the expression for the black hole entropy. Moreover, in [35], it was shown that this symmetry-based approach can be further used to calculate the logarithmic correction to the BTZ black hole and it was found that $\mathbf{C}=-3 / 2$. $^{2}$

Importantly, the above procedure can also be generalized to compute logarithmic corrections to the entropy of hyperbolic black holes which are relevant for our purpose here, especially since it is unclear (at-least to the author) how to calculate logarithmic corrections to entropy of these black holes in LQG or in string theory context. In this paper, using the methodology of [36], we calculate these corrections for two gravity theories, namely Einstein and Gauss-Bonnet gravity. We find $\mathbf{C}=-3 / 2$ for both cases. However, we want to stress from the outset that the whole purpose for this exercise is to show at-least one method by which corrections to the entropy of the hyperbolic black holes can be explicitly cal-

\footnotetext{
${ }^{1}$ Black hole entropy can also acquires logarithmic corrections due to thermal fluctuations in the black hole extensive parameters. More details can be found in [23-27].

2 This result was based on certain assumptions. We will discuss these assumptions in more detail in Sect. 4.
} 
culated and that it is non-zero. Although important in its own way, it is not our main objective to settle down the controversial nature of $\mathbf{C}$ here. Instead, we want to probe the effects of this $\mathbf{C}$ on the Rényi entropy. For this reason, we will work with arbitrary $\mathbf{C}$ in this paper for most of the time.

Using the corrected expression for the entropy of the black hole (Eq. (4)) into the prescription of [8,10], we find that there are corrections to the standard results of Rényi entropies. These corrections are both logarithmic and non-logarithmic in nature. For a two-dimensional CFT, we find that the correction terms in the Rényi entropy are a function of the logarithmic of the central charge as well as index $q$. However, the dependence on the size of the system is doubly logarithmic. ${ }^{3}$ For higher dimension CFTs, Rényi entropy is a complicated function of $q$. For the entanglement entropy our results simplify tremendously. We find that correction term in the entanglement entropy is proportional to $\mathbf{C}$ times the logarithm of its standard expression. Interestingly, at the order $G_{D}^{0}$, the correction term in the entanglement entropy depends logarithmically on the area of the spherical entangling surface. This is important since quantum corrections to the holographic entanglement entropy are expected to be of order $G_{D}^{0}$. Moreover, using the renormalized entanglement entropy prescription of [37], we find that the corrected entanglement entropy expression can again be properly renormalized and that the universal part of the entanglement entropy remains the same even with the correction terms. Therefore, the entropic $c$ theorem is again satisfied. Our analysis further suggests a similar kind of corrections in the Rényi entropy too. With a Gauss-Bonnet black hole as a gravity dual, the holographic Rényi entropy is found to be a complicated function of two distinct central charges. However, the entanglement entropy depends only on one central charge. The correction terms in the entanglement entropy are again found to be proportional to $\mathbf{C}$ times the logarithm of its standard expression.

It is also well known that the Rényi entropy satisfies a few inequalities involving the derivative with respect to $q[38,39]$. We find that, for higher-dimensional CFTs, these inequalities are again satisfied even with correction terms provided the coefficient $\mathbf{C}$ is not very large. However, for two-dimensional CFTs, a few of these inequalities can be violated in the limit of small central charge. However, for large central charge, which is the case for the boundary CFT with gravity dual, these inequalities are again found to be satisfied. Our analysis therefore provide a strong evidence for their validity at order $G_{D}^{0}$.

The RT prescription only provides the leading order result of the EE (which is of order $N^{2}$ or $G_{D}^{-1}$ ) and much attention now a days has been paid to obtained the subleading corrections to it (of the order of $G_{D}^{0}$ ). Recently some work

\footnotetext{
${ }^{3}$ Similar kind of doubly logarithmic structure in the entanglement entropy has also appeared in a different system in [33].
}

has been done in this direction; see for example [40,41]. In [40], it was conjectured that the subleading correction to the entanglement entropy is given by the entanglement of the Ryu-Takayanagi minimal-area surface with the rest of the bulk AdS. This again requires the difficult replica method in the bulk AdS to obtained the subleading correction to the entanglement entropy. Moreover, this conjecture for the subleading order is for the entanglement entropy only and no such analogous conjecture is known for the Rényi entropy. In [41], subleading corrections to the entanglement and Rényi entropies were obtained by calculating the one-loop determinants around the classical solutions using the Schottky uniformization of q-sheeted Riemann surface. There are again many difficulties and limitations in implementing this method, such as that constructing the smooth bulk solutions is not always straightforward and that performing analytic continuation of the replica index to non-integer $\mathrm{q}$ is sometimes very difficult. One can use the method of [41] to calculate the corrections for $1+1$-dimensional CFT with Einstein gravity in the bulk. Whether this method can be generalized for higher-dimensional conformal field theory or to higher derivative gravity theory in the bulk is not clear yet.

In this work we are suggesting another method by which not only the corrections to entanglement entropy but also to the Rényi entropies can be obtained. Our idea does not involve the mathematical complexity of $[40,41]$ and is easy to implement. This method again has the limitation that it works only for the spherical entangling surface.

The paper is organized as follows: In the next section, we review the main ideas of $[8,10]$ to relate the Rényi entropy to the entropy of the black hole. In Sect. 3, we highlight the necessary steps to calculate the asymptotic form of density of states using the two-dimensional conformal algebra. In Sect. 4, we first construct the Virasoro algebra having a central extension on the black hole horizon and then use the expression of the density of states to calculate the the entropy of the black hole. In the process we calculate the logarithmic correction to the entropy of AdS-Schwarzschild and GaussBonnet black hole. In Sect. 5, we analyze the holographic Rényi entropy in detail and discuss the nature of the correction terms. Finally, we conclude by summarizing our main results in Sect. 6.

\section{Holographic entanglement and Rényi entropies}

In this section, we review some aspects of holographic entanglement and Rényi entropies which will be the focus of this paper. As mentioned in the introduction, we follow the prescription of $[8,10]$ to calculate these entropies for a spherical entangling region in the boundary CFT using the AdS/CFT correspondence. Let us first briefly discuss the work done in these papers to set the stage. 
Consider a $d$-dimensional CFT on Minkowski space and choose a spherical entangling surface of radius $R$ as a subsystem. The computation of entanglement entropy of this subsystem with the rest of the system can be performed by calculating the reduced density matrix $\rho_{v}$. However, the authors in [8], using the conformal structure of the theory, mapped this problem of the entanglement entropy to the thermal entropy on a hyperbolic space $R \times H^{d-1}$. They showed that the causal development of the ball enclosed by the spherical entangling surface can be mapped to a hyperbolic space $R \times H^{d-1}$; with curvature of $H^{d-1}$ space given by the radius of the spherical entangling region $R$. An important point of this mapping was that the vacuum of the original CFT mapped to a thermal bath with temperature

$T_{0}=\frac{1}{2 \pi R}$

on the hyperbolic space. Now relating the density matrix $\rho_{\text {therm }}$ in the new spacetime $R \times H^{d-1}$ to the old spacetime $\rho_{v}$ by a unitary transformation, $\rho_{v}=U^{-1} \rho_{\text {therm }} U$, we get

$\rho_{v}=U^{-1} \frac{e^{\left[-H / T_{0}\right]}}{Z\left(T_{0}\right)} U$,

where $Z\left(T_{0}\right)=\operatorname{Tr}\left[e^{-H / T_{0}}\right]$. For the Rényi entropy we also need the $q$ th power of $\rho_{v}$. From the above equation, we get

$\rho_{v}^{q}=U^{-1} \frac{e^{\left[-q H / T_{0}\right]}}{Z\left(T_{0}\right)^{q}} U$.

Taking the trace of both sides of Eq. (7), we get

$\operatorname{Tr}\left[\rho_{v}^{q}\right]=\frac{Z\left(T_{0} / q\right)}{Z\left(T_{0}\right)^{q}}$,

as $U$ and its inverse cancel each other upon taking trace. Now, using the definition of Rényi entropy as in Eq. (2), we arrive at

$S_{q}=\frac{1}{1-q}\left[\ln Z\left(T_{0} / q\right)-q \ln Z\left(T_{0}\right)\right]$.

The above expression for the Rényi entropy can also be written in terms of the free energy $F(T)=-T \ln Z(T)$ :

$S_{q}=\frac{q}{1-q} \frac{1}{T_{0}}\left[F\left(T_{0}\right)-F\left(T_{0} / q\right)\right]$

and further, using the thermodynamic relation $S_{\text {therm }}=$ $-\partial F / \partial T$, we can rewrite the above expression as

$S_{q}=\frac{q}{q-1} \frac{1}{T_{0}} \int_{T_{0} / q}^{T_{0}} \mathrm{~d} T S_{\text {therm }}(T) ;$

here, just to clarify again, $S_{\text {therm }}$ is the thermal entropy of a $d$-dimensional CFT on $R \times H^{d-1}$, while $S_{q}$ is the desired Rényi entropy. Equation (11) was the main result of $[8,10]$, which relates Rényi (and hence entanglement) entropy of a spherical entangling region in $d$-dimensional CFT to the thermal entropy on a hyperbolic space. As pointed out in
[8], the above analysis just mapped one difficult problem to another equally difficult problem and is not particularly useful for practical purposes. However, its true usefulness can be realized via the AdS/CFT correspondence. In the AdS/CFT correspondence, the thermal state of the boundary CFT corresponds to an appropriate non-extremal black hole in the bulk AdS spacetime, with thermal entropy corresponding to black hole entropy. Therefore, using the AdS/CFT correspondence, we can relate $S_{\text {therm }}$ appearing in Eq. (11) to that of the black hole entropy, which is relatively easy to compute. Since on the boundary side our CFT is on $R \times H^{d-1}$, its dual gravity theory will be described by a topological black hole with hyperbolic event horizon. In any event, in this AdS/CFT approach, the Rényi entropy is now given by the horizon entropy of the corresponding hyperbolic black hole, which can easily be computed using Ward's standard formula. However, since we are interested in calculating the effects of the corrections of the entropy of the black hole on the Rényi entropy, here we will use an approach based on symmetry arguments on the horizon to calculate the black hole entropy, instead of Wald's formula. This is the topic of the discussion of the next section.

\section{Logarithmic corrections to the entropy of the black hole from the Cardy formula}

In this section, we will describe the necessary steps to calculate the asymptotic form of density of states from a twodimensional conformal algebra. ${ }^{4}$ This form of density of states will be used in a later section to calculate the black hole entropy and, further, to compute logarithmic corrections. In this section, we will mostly follow the notations used in [35] and refer the reader to [35] for a detailed discussion. ${ }^{5}$

We start with a standard Virasoro algebra of the two conformal field theory with central charges $c, \bar{c}$ :

$$
\begin{aligned}
& {\left[L_{m}, L_{n}\right]=(m-n) L_{m+n}+\frac{c}{12} m\left(m^{2}-1\right) \delta_{m+n, 0}} \\
& {\left[\bar{L}_{m}, \bar{L}_{n}\right]=(m-n) \bar{L}_{m+n}+\frac{\bar{c}}{12} m\left(m^{2}-1\right) \delta_{m+n, 0}} \\
& {\left[L_{m}, \bar{L}_{n}\right]=0}
\end{aligned}
$$

here $L_{m}$ and $\bar{L}_{m}$ are the generators of holomorphic and antiholomorphic diffeomorphisms. If $\rho(\Delta, \bar{\Delta})$ denotes the degeneracy of states carrying $L_{0}=\Delta$ and $\bar{L}_{0}=\bar{\Delta}$ eigenvalues, then one can define the partition function on the twotorus of modulus $\tau=\tau_{1}+i \tau_{2}$ as

\footnotetext{
${ }^{4}$ See [42], for generalization of the Cardy formula in higherdimensional CFT.

5 Extension of the Cardy formula beyond the subleading order was performed in [43].
} 


$$
\begin{aligned}
& Z(\tau, \bar{\tau})=\operatorname{Tr}\left(e^{2 \pi i \tau L_{0}} e^{-2 \pi i \bar{\tau} \bar{L}_{0}}\right) \\
& =\sum_{\Delta, \bar{\Delta}}\left(\rho(\Delta, \bar{\Delta}) e^{2 \pi i \tau \Delta} e^{-2 \pi i \bar{\tau} \bar{\Delta}}\right) .
\end{aligned}
$$

Now using $q=e^{2 \pi i \tau}, \bar{q}=e^{-2 \pi i \bar{\tau}}$ and inverting the above equation, we get

$\rho(\Delta, \bar{\Delta})=\frac{1}{(2 \pi i)^{2}} \int \frac{1}{q^{\Delta+1}} \frac{1}{\bar{q}^{\bar{\Delta}+1}} Z(q, \bar{q}) \mathrm{d} q \mathrm{~d} \bar{q}$,

where the integrals are along contours that enclose $q=0$ and $\bar{q}=0$. Therefore, if we know the partition function $Z(q, \bar{q})$, then we can use Eq. (14) to determine the density of states. Now modular invariance of the theory implies that

$Z_{0}(\tau, \bar{\tau})=\operatorname{Tr}\left[e^{2 \pi i \tau\left(L_{0}-\frac{c}{24}\right)} e^{-2 \pi i \bar{\tau}\left(\bar{L}_{0}-\frac{\bar{c}}{24}\right)}\right]$.

$Z_{0}$ is invariant under large $\tau \longrightarrow-1 / \tau$ diffeomorphism. Cardy has shown that the invariance in Eq. (15) is based on the general properties of two-dimensional conformal field theory and therefore expected to be universal. From Eqs. (13) and (15), we note that

$Z(\tau, \bar{\tau})=e^{\frac{2 \pi i c \tau}{24}} e^{-\frac{2 \pi i \bar{c} \bar{\tau}}{24}} Z_{0}(\tau, \bar{\tau})$

using the modular invariance of $Z_{0}$, we get

$Z(\tau, \bar{\tau})=e^{\frac{2 \pi i c}{24}\left(\tau+\frac{1}{\tau}\right)} e^{-\frac{2 \pi i \bar{c}}{24}\left(\bar{\tau}+\frac{1}{\bar{\tau}}\right)} Z\left(-\frac{1}{\tau},-\frac{1}{\bar{\tau}}\right)$,

substituting Eq. (16) into Eq. (14), we obtain

$$
\begin{aligned}
\rho(\Delta, \bar{\Delta})= & \int \mathrm{d} \tau \mathrm{d} \bar{\tau} e^{-2 \pi i \Delta \tau+\frac{2 \pi i c}{24}\left(\tau+\frac{1}{\tau}\right)} e^{2 \pi i \bar{\Delta} \bar{\tau}-\frac{2 \pi i \bar{c}}{24}\left(\bar{\tau}+\frac{1}{\bar{\tau}}\right)} \\
& \times Z\left(-\frac{1}{\tau},-\frac{1}{\bar{\tau}}\right) .
\end{aligned}
$$

The above integral has the form

$I[a, b]=\int \mathrm{d} \tau e^{2 \pi i a \tau+\frac{2 \pi i b}{\tau}} \mathcal{F}(\tau)$,

which can be evaluated by saddle point approximation. For this purpose, we need to assume that $\mathcal{F}(\tau)$ is slowly varying near the extremum of the phase. As shown in [35], this is indeed the case if one considers the situation where the imaginary part of $\tau$ is large. Now the saddle point, obtained by extremizing the exponent on the right hand side of the above equation, is at $\tau_{0}=\sqrt{b / a} \approx i \sqrt{c / 24 \Delta}$, where we have assumed a large $\Delta$. Expanding around this saddle point, we find

$$
\begin{gathered}
I[a, b] \approx \int \mathrm{d} \tau e^{4 \pi i \sqrt{a b}+\frac{2 \pi i b}{\tau_{0}^{3}}\left(\tau-\tau_{0}\right)^{2}} \mathcal{F}\left(\tau_{0}\right) \\
=\left(-\frac{b}{4 a^{3}}\right)^{1 / 4} e^{4 \pi i \sqrt{a b}} \mathcal{F}\left(\tau_{0}\right),
\end{gathered}
$$

and an analogous expression exists for the $\bar{\tau}$ integral. Finally, one obtains the expression for the density of states as

$\rho(\Delta, \bar{\Delta}) \approx\left(\frac{c}{96 \Delta^{3}}\right)^{\frac{1}{4}}\left(\frac{\bar{c}}{96 \bar{\Delta}^{3}}\right)^{\frac{1}{4}} e^{2 \pi \sqrt{\frac{c \Delta}{6}}} e^{2 \pi \sqrt{\frac{\bar{c} \bar{\Delta}}{6}}}$.

Since, for most cases of our interest, we have only one Virasoro algebra instead of two (see the next section for details), the relevant expression for density of states is

$\rho(\Delta) \approx\left(\frac{c}{96 \Delta^{3}}\right)^{\frac{1}{4}} e^{2 \pi \sqrt{\frac{c \Delta}{6}}}$.

In the above expression, the exponential term is the standard Cardy formula. However, an important part, which will play a significant role in our analysis later on, is the term that has a power law behavior. In the next section, we will use the logarithm of $\rho$ to calculate the entropy of the black holes. As one can anticipate, the exponential part of Eq. (21) will give the usual Wald entropy and, on the other hand, the power term will provide a logarithmic correction to the entropy of the black hole.

\section{Black hole entropy and Virasoro algebra from the surface term of the gravitational action}

There has been a lot of activity in understanding the black hole entropy using the symmetry-based horizon CFT approach. This approach essentially assumes that the symmetries of a black hole horizon are sufficient to compute the density of states (hence the black hole entropy) at a given energy. Many avatars of this approach have appeared in the literature, and all of them have successfully predicted the expression for the entropy of the black hole. ${ }^{6}$ A complete list of references for later developments can be found in [44].

Applying a similar line of symmetry reasoning, a new approach was recently proposed in [36] which is straightforward and conceptually clearer. Importantly, it does not require any ad hoc prescription such as shifting the zeromode energy and is also easy to implement. In this approach, the Noether currents associated with the diffeomorphism invariance of the Gibbons-Hawking boundary action, instead of the bulk gravity action, are used to construct the Virasoro algebra at the horizon. Here, the diffeomorphisms are chosen in such a way that they leave the near-horizon structure of the metric invariant. The Virasoro algebra constructed in this way is again found to have a central extension, which upon using the Cardy formula correctly reproduces the black hole entropy expression. In this work, we will follow this

\footnotetext{
${ }^{6}$ A certain level of arbitrariness is present in the procedure of all symmetry-based approaches in order to produce the correct black hole entropy expression. We will further discuss this arbitrariness in the following.
} 
boundary Noether current procedure of [36] to calculate the black hole entropy. We will first discuss its general formalism and then apply this formalism to calculate the entropy of the black hole of two gravity theories, namely Einstein and Gauss-Bonnet gravity.

We start with the Gibbons-Hawking surface term $I_{\mathrm{GH}}$ :

$$
\begin{aligned}
I_{\mathrm{GH}}= & \frac{1}{8 \pi G_{D}} \int_{\partial \mathcal{M}} d^{D-1} x \sqrt{-\gamma} \mathfrak{L}=\frac{1}{8 \pi G_{D}} \\
& \times \int_{\mathcal{M}} d^{D} x \sqrt{-g} \nabla_{a}\left(n^{a} \mathfrak{L}\right),
\end{aligned}
$$

where $\partial \mathcal{M}$ is the boundary of the manifold $\mathcal{M}, \mathfrak{L}$ is related to the trace of the extrinsic curvature, $n^{a}$ is the unit normal vector to the boundary, $g_{\mu \nu}$ denotes the bulk metric and $\gamma_{\mu \nu}$ is the induced metric on the boundary. The expression for $\mathfrak{L}$ depends on the gravity theory under consideration. For example, in Einstein gravity it is equal to trace of the extrinsic curvature $\mathcal{K}=-\nabla_{\mu} n^{\mu}$. The conserved current $J^{\mu}$ associated with the diffeomorphism invariance $x^{\mu} \longrightarrow x^{\mu}+\xi^{\mu}$ of $I_{\mathrm{GH}}$ can be obtained by considering the variation of both sides of Eq. (22) as the Lie derivative. After a bit of algebra, one gets

$J^{\mu}[\xi]=\nabla_{\nu} J^{\mu \nu}[\xi]=\frac{1}{8 \pi G_{D}} \nabla_{\nu}\left(\mathfrak{L} \xi^{\mu} n^{\nu}-\mathfrak{L} \xi^{\nu} n^{\mu}\right)$.

The charge corresponding to this conserved current is defined as

$Q[\xi]=\int_{\partial \Sigma} \mathrm{d} \Sigma_{\mu \nu}\left(\sqrt{-h} J^{\mu \nu}\right)$,

where $J^{\mu \nu}$ is called the Noether potential and $\mathrm{d} \Sigma_{\mu \nu}=$ $-d^{D-2} x\left(n_{\mu} m_{v}-m_{\mu} n_{v}\right)$ is the surface element of the $(D-2)$-dimensional surface $\partial \Sigma$ with metric $h_{\mu \nu}$. In order to discuss black hole physics, we will choose the surface $\partial \Sigma$ to be near the horizon. The unit vectors $n^{\mu}$ and $m^{\mu}$ are chosen to be spacelike and timelike, respectively.

Following [36], we define the Lie bracket of the charges as

$$
\begin{aligned}
{\left[Q\left[\xi_{1}\right], Q\left[\xi_{2}\right]\right] } & \equiv\left(\delta_{\xi_{1}} Q\left[\xi_{2}\right]-\delta_{\xi_{2}} Q\left[\xi_{1}\right]\right) \\
& =\int_{\partial \Sigma} \mathrm{d} \Sigma_{\mu \nu} \sqrt{-h}\left(\xi_{2}^{\mu} J^{\nu}\left[\xi_{1}\right]-\xi_{1}^{\mu} J^{\nu}\left[\xi_{2}\right]\right) .
\end{aligned}
$$

From the above equation, it is clear that we only need to know the vector field $\xi^{\mu}$ to determine the charge algebra. This can be done, as explained below, by choosing an appropriate diffeomorphism which leaves the horizon structure invariant. However, in order to proceed, let us first write the $D(=d+1)$ dimensional form of the metric as

$\mathrm{d} s^{2}=-f(r) N^{2} \mathrm{~d} t^{2}+\frac{\mathrm{d} r^{2}}{f(r)}+r^{2} \mathrm{~d} \Omega_{i j}(x) \mathrm{d} x^{i} \mathrm{~d} x^{j}$,

where $\mathrm{d} \Omega_{i j}(x) \mathrm{d} x^{i} \mathrm{~d} x^{j}$ is the line element of the $(d-1)$ dimensional space. For our discussion in the next section, we will take this line element to be hyperbolic but as of now this metric is completely general. The constant $N^{2}$ is included in $g_{t t}$ to allow us to adjust the normalization of the time coordinate. This will be useful later on, but will not play any significant role in the black hole entropy calculation here. To study the near-horizon structure, defined by $f\left(r_{h}\right)=0$, it is convenient to choose a coordinate $r=\tilde{r}+r_{h}$, in which case the above metric reduces to

$$
\begin{aligned}
\mathrm{d} s^{2}= & -f\left(\tilde{r}+r_{h}\right) N^{2} \mathrm{~d} t^{2}+\frac{\mathrm{d} \tilde{r}^{2}}{f\left(\tilde{r}+r_{h}\right)} \\
& +\left(\tilde{r}+r_{h}\right)^{2} \mathrm{~d} \Omega_{i j}(x) \mathrm{d} x^{i} \mathrm{~d} x^{j} .
\end{aligned}
$$

In the near-horizon region i.e. $\tilde{r} \rightarrow 0$ limit, the function $f\left(\tilde{r}+r_{h}\right)$ can be expanded as

$f\left(\tilde{r}+r_{h}\right)=\tilde{r} f^{\prime}\left(r_{h}\right)+1 / 2 \tilde{r}^{2} f^{\prime \prime}\left(r_{h}\right)+\cdots$

Defining the surface gravity $\kappa$ as $\kappa=N f^{\prime}\left(r_{h}\right) / 2$ and dropping the terms beyond first order in $f\left(\tilde{r}+r_{h}\right)$, one notice that the $(t-\tilde{r})$ part of the metric in Eq. (27) reduces to the standard Rindler metric

$\mathrm{d} s_{\tilde{t}-\tilde{r}}^{2}=-2 \tilde{r} \frac{\kappa}{N} \mathrm{~d} \tilde{t}^{2}+\frac{1}{2 \tilde{r}} \frac{N}{\kappa} \mathrm{d} \tilde{r}^{2}$,

where $\tilde{t}=N t$. The unit normal vectors can be chosen as

$$
\begin{aligned}
n^{\mu} & =\left(0, \sqrt{f\left(\tilde{r}+r_{h}\right)}, 0, \ldots, 0\right), \quad m^{\mu} \\
& =\left(1 / N \sqrt{f\left(\tilde{r}+r_{h}\right)}, 0,0, \ldots, 0\right)
\end{aligned}
$$

Further, in order to find $\xi^{\mu}$, it is more useful to first transform to Bondi like coordinates by the transformation

$\mathrm{d} u=\mathrm{d} \tilde{t}-\frac{\mathrm{d} \tilde{r}}{f\left(\tilde{r}+r_{h}\right)}$

under which the metric in Eq. (27) reduces to

$\mathrm{d} s^{2}=-f\left(\tilde{r}+r_{h}\right) \mathrm{d} u^{2}-2 \mathrm{~d} u \mathrm{~d} \tilde{r}+\left(\tilde{r}+r_{h}\right)^{2} \mathrm{~d} \Omega_{i j}(x) \mathrm{d} x^{i} \mathrm{~d} x^{j}$.

Now we choose our vector field $\xi^{\mu}$ by imposing the condition that the horizon structure remain invariant under diffeomorphism i.e. the metric coefficients $g_{\tilde{r} \tilde{r}}$ and $g_{u \tilde{r}}$ remain unchanged. This implies the following Killing equations, ${ }^{7}$ :

$$
\begin{aligned}
& \mathcal{L}_{\xi} g_{\tilde{r} \tilde{r}}=-2 \partial_{\tilde{r}} \xi^{u}=0 \\
& \mathcal{L}_{\xi} g_{u \tilde{r}}=-f\left(\tilde{r}+r_{h}\right) \partial_{\tilde{r}} \xi^{u}-\partial_{u} \xi^{u}-\partial_{\tilde{r}} \xi^{\tilde{r}}=0 .
\end{aligned}
$$

Solving the above two equations, we get

$\xi^{u}=\mathcal{S}(u, \vec{x}), \quad \xi^{\tilde{r}}=-\tilde{r} \partial_{u} \mathcal{S}(u, \vec{x})$,

\footnotetext{
$\overline{7}$ It is easy to check that the other condition $\mathcal{L}_{\xi} g_{u u}=0$ is trivially satisfied near the horizon.
} 
where $\mathcal{S}$ is an arbitrary function and $\vec{x}$ are the coordinates on the remaining $(d-1)$-dimensional space. Now converting back to the $(t, \tilde{r})$ coordinates, these vector fields take the form

$\xi^{t}=T-\frac{\tilde{r}}{N f\left(\tilde{r}+r_{h}\right)} \partial_{t} T, \quad \xi^{\tilde{r}}=-\frac{\tilde{r}}{N} \partial_{t} T$,

where $T(t, \tilde{r}, \vec{x}) \equiv \mathcal{S}(u, \vec{x})$. Therefore, we can evaluate the expressions for $\xi^{\mu}, Q\left[\xi^{\mu}\right]$ and the charge algebra $\left[Q\left[\xi_{1}\right], Q\left[\xi_{2}\right]\right]$ once the function $T$ is given. Now, we expand this function $T$ in terms of a set of basis functions $T_{m}$, as

$T=\sum_{m} A_{m} T_{m}, \quad A_{m}^{*}=A_{-m}$.

As is standard in the literature, we choose $T_{m}$ such that the resulting $\xi_{m}^{\mu}$ obeys the algebra isomorphic to Diff $S^{1}$ i.e.

$i\left\{\xi_{m}, \xi_{n}\right\}^{\mu}=(m-n) \xi_{m+n}^{\mu}$,

where $\{$,$\} is the Lie bracket. A particular choice is$

$T_{m}=\frac{1}{\alpha} e^{i m(\alpha t+g(\tilde{r})+\vec{p} \cdot \vec{x})}$,

where $\alpha$ is an arbitrary parameter, $g(\tilde{r})$ is a function which is regular at the horizon and $p_{i}$ are integers. We now have all the ingredients at our disposal to calculate the leading Wald as well as the subleading logarithmic correction to the entropy of the black hole. From now on we will concentrate on horizons with hyperbolic topology, as this will be required in the computation of the holographic Rényi entropy in the later section.

Before going to discuss the entropy of the black hole explicitly, we like to clarify that the Noether current of Eq. (23) is associated only with the diffeomorphism invariance of the surface term and should not be confused with the usual Noether current which is associated with the bulk gravity Lagrangian [45]. The surface term here is used just to construct the relevant Virasoro algebra, which we then evaluate on the static horizon. Once we have the Virasoro algebra the black hole entropy follows from the Cardy formula. Thus the surface term is not directly contributing to the entropy of the black hole, and therefore, not contradicting [45].

\subsection{Entropy of AdS-Schwarzschild Black hole}

We first apply the formalism developed above to a $(d+1)$ dimensional AdS-Schwarzschild black hole in Einstein gravity. As mentioned earlier, for Einstein gravity the GibbonsHawking surface term is standard and is given by the expression $\mathfrak{L}=\mathcal{K}=-\nabla_{\mu} n^{\mu}$. The metric is given as

$$
\begin{aligned}
& \mathrm{d} s^{2}=-f(r) N^{2} \mathrm{~d} t^{2}+\frac{1}{f(r)} \mathrm{d} r^{2}+r^{2} \mathrm{~d} \Sigma_{d-1}^{2}, \\
& f(r)=-1-\frac{m}{r^{d-2}}+\frac{r^{2}}{L^{2}},
\end{aligned}
$$

where

$\mathrm{d} \Sigma_{d-1}^{2}=\mathrm{d} \theta^{2}+\sinh ^{2} \theta \mathrm{d} \Omega_{d-2}^{2}$

is the metric on the $(d-1)$-dimensional hyperbolic space with $\mathrm{d} \Omega_{d-2}^{2}$ being the line element on a unit $(d-2)$-sphere. The exact value of constant $N^{2}$ is not required here and will be specified in the next section. Substituting Eq. (37) into Eqs. (33), (23), (24) and (25) and taking the near-horizon limit $\tilde{r} \rightarrow 0$, we get

$Q\left[\xi_{m}\right]=\frac{1}{8 \pi G_{D}} \int_{\mathcal{H}} d^{d-1} x \sqrt{h}\left(\kappa T_{m}-\frac{1}{2} \partial_{t} T_{m}\right)$.

Similarly, the algebra of the charges corresponding to $T=T_{m}$ is given by

$$
\begin{array}{r}
{\left[Q\left[\xi_{m}\right], Q\left[\xi_{n}\right]\right]=\frac{1}{8 \pi G_{D}} \int_{\mathcal{H}} d^{d-1} x \sqrt{h}\left[\kappa\left(T_{m} \partial_{t} T_{n}-T_{n} \partial_{t} T_{m}\right)\right.} \\
\left.-\frac{1}{2}\left(T_{m} \partial_{t}^{2} T_{n}-T_{n} \partial_{t}^{2} T_{m}\right)+\frac{1}{4 \kappa}\left(\partial_{t} T_{m} \partial_{t}^{2} T_{n}-\partial_{t} T_{n} \partial_{t}^{2} T_{m}\right)\right] .
\end{array}
$$

Now substituting Eqs. (36) and (37) into Eqs. (39) and (40), we get the final expressions as

$$
\begin{aligned}
& Q\left[\xi_{m}\right]=\frac{A_{d-1}}{8 \pi G_{D}} \frac{\kappa}{\alpha} \delta_{m, 0} \\
& {\left[Q\left[\xi_{m}\right], Q\left[\xi_{n}\right]\right]=-i(m-n) \frac{A_{d-1}}{8 \pi G_{D}} \frac{\kappa}{\alpha} \delta_{m+n, 0}} \\
& -i m^{3} \frac{A_{d-1}}{16 \pi G_{D}} \frac{\alpha}{\kappa} \delta_{m+n, 0},
\end{aligned}
$$

where $A_{d-1}$ is the area of $(d-1)$-dimensional hyperbolic space (the horizon area). Strikingly, the above charge algebra is quite similar to the two dimensional Virasoro algebra discussed in the previous section. From the above expressions, we obtain the central term in the algebra as

$$
\begin{aligned}
K\left[\xi_{m}, \xi_{n}\right] & =\left[Q\left[\xi_{m}\right], Q\left[\xi_{n}\right]\right]+i(m-n) Q\left[\xi_{m+n}\right] \\
& =-i m^{3} \frac{A_{d-1}}{16 \pi G_{D}} \frac{\alpha}{\kappa} \delta_{m+n, 0},
\end{aligned}
$$

from which we can read off the zero mode $Q_{0}$ and central charge $c$ as

$$
Q_{0}=Q\left[\xi_{0}\right]=\frac{A_{d-1}}{8 \pi G_{D}} \frac{\kappa}{\alpha}, \quad \frac{c}{12}=\frac{A_{d-1}}{16 \pi G_{D}} \frac{\alpha}{\kappa} .
$$

Substituting above expressions into Eq. (21) with $Q_{0}=\Delta$ and taking log on both side, we obtain the black hole entropy

$$
\begin{aligned}
& S=\ln \rho \approx 2 \pi \sqrt{\frac{c \Delta}{6}}+\frac{1}{4} \ln \frac{c}{\Delta^{3}}+\cdots \\
& S=\frac{A_{d-1}}{4 G_{D}}-\frac{3}{2} \ln \frac{A_{d-1}}{4 G_{D}}+\cdots=S_{\text {Wald }}-\frac{3}{2} \ln S_{\text {Wald }}+\cdots
\end{aligned}
$$


We see that the leading term matches exactly with the usual black hole entropy expression. We also find a logarithmic correction to it. Interestingly, the coefficient $-3 / 2$ of the logarithmic correction for the hyperbolic horizon is the same as was found for the BTZ black hole in [35]. In order to find this coefficient we have chosen the arbitrary parameter $\alpha$ to be such that the central charge $c$ is a universal constant i.e. independent of the area $A_{d-1}$, as in [35]. We will say more about this condition and the coefficient of this logarithmic term at the end of this section.

\subsection{Entropy of Gauss-Bonnet black hole}

Now we move on to discuss the entropy of Gauss-Bonnet black holes. The surface term of the gravitational action can be found in [46-49] and is given as

$\mathfrak{L}=-\nabla_{\mu} n^{\mu}+\frac{2 \lambda L^{2}}{(D-3)(D-4)}\left(P-2 \tilde{G}_{\mu \nu} \mathcal{K}^{\mu \nu}\right)$,

where $\lambda$ is the coefficient of the Gauss-Bonnet term, $P$ is the trace of the tensor

$P_{\mu \nu}=\frac{1}{3}\left(2 \mathcal{K} \mathcal{K}_{\mu \rho} \mathcal{K}^{\rho}{ }_{\nu}+\mathcal{K}_{\rho \sigma} \mathcal{K}^{\rho \sigma} \mathcal{K}_{\mu \nu}-2 \mathcal{K}_{\mu \rho} \mathcal{K}^{\rho \sigma} \mathcal{K}_{\sigma \nu}-\mathcal{K}^{2} \mathcal{K}_{\mu \nu}\right)$

and $\tilde{G}_{\mu \nu}$ stands for Einstein tensor of $d$-dimensional boundary metric. For a Gauss-Bonnet black hole, the $D$-dimensional metric is given by

$$
\begin{gathered}
\mathrm{d} s^{2}=-f(r) N^{2} \mathrm{~d} t^{2}+\frac{1}{f(r)} \mathrm{d} r^{2}+r^{2} \mathrm{~d} \Sigma_{d-1}^{2}, \\
f(r)=-1+\frac{r^{2}}{2 \lambda L^{2}}\left[1-\sqrt{1-4 \lambda+\frac{4 \lambda m}{r^{D-1}}}\right],
\end{gathered}
$$

where $m$ is related to the mass of the black hole. Performing the analogous steps as in the previous subsection to calculate the charge and Virasoro algebra for Gauss-Bonnet black hole, we get

$$
\begin{aligned}
& Q\left[\xi_{m}\right]=\frac{1}{8 \pi G_{D}}\left(1-\frac{2(D-2) \lambda L^{2}}{(D-4) r_{h}^{2}}\right) \\
& \times \int_{\mathcal{H}} d^{d-1} x \sqrt{h}\left(\kappa T_{m}-\frac{1}{2} \partial_{t} T_{m}\right) \\
& {\left[Q\left[\xi_{m}\right], Q\left[\xi_{n}\right]\right]=\frac{1}{8 \pi G_{D}}\left(1-\frac{2(D-2) \lambda L^{2}}{(D-4) r_{h}^{2}}\right)} \\
& \times \int_{\mathcal{H}} d^{d-1} x \sqrt{h}\left[\kappa\left(T_{m} \partial_{t} T_{n}-T_{n} \partial_{t} T_{m}\right)\right. \\
& \left.-\frac{1}{2}\left(T_{m} \partial_{t}^{2} T_{n}-T_{n} \partial_{t}^{2} T_{m}\right)+\frac{1}{4 \kappa}\left(\partial_{t} T_{m} \partial_{t}^{2} T_{n}-\partial_{t} T_{n} \partial_{t}^{2} T_{m}\right)\right]
\end{aligned}
$$

Now substituting Eqs. (36) and (47) into Eqs. (48) and (49), we obtain

$Q\left[\xi_{m}\right]=\frac{A_{d-1}}{8 \pi G_{D}}\left(1-\frac{2(D-2) \lambda L^{2}}{(D-4) r_{h}^{2}}\right) \frac{\kappa}{\alpha} \delta_{m, 0}$

$\left[Q\left[\xi_{m}\right], Q\left[\xi_{n}\right]\right]$

$$
\begin{aligned}
= & -i(m-n) \frac{A_{d-1}}{8 \pi G_{D}} \frac{\kappa}{\alpha}\left(1-\frac{2(D-2) \lambda L^{2}}{(D-4) r_{h}^{2}}\right) \delta_{m+n, 0} \\
& -i m^{3} \frac{A_{d-1}}{16 \pi G_{D}} \frac{\alpha}{\kappa}\left(1-\frac{2(D-2) \lambda L^{2}}{(D-4) r_{h}^{2}}\right) \delta_{m+n, 0},
\end{aligned}
$$

from which, we obtain the central term

$$
\begin{aligned}
K\left[\xi_{m}, \xi_{n}\right] & =\left[Q\left[\xi_{m}\right], Q\left[\xi_{n}\right]\right]+i(m-n) Q\left[\xi_{m+n}\right] \\
& =-i m^{3} \frac{A_{d-1}}{16 \pi G_{D}} \frac{\alpha}{\kappa}\left(1-\frac{2(D-2) \lambda L^{2}}{(D-4) r_{h}^{2}}\right) \delta_{m+n, 0},
\end{aligned}
$$

and we can read off the zero mode $Q_{0}$ and central charge $c$ as

$$
\begin{aligned}
Q_{0} & =\frac{A_{d-1}}{8 \pi G_{D}} \frac{\kappa}{\alpha}\left(1-\frac{2(D-2) \lambda L^{2}}{(D-4) r_{h}^{2}}\right), \\
\frac{c}{12} & =\frac{A_{d-1}}{16 \pi G_{D}} \frac{\alpha}{\kappa}\left(1-\frac{2(D-2) \lambda L^{2}}{(D-4) r_{h}^{2}}\right) .
\end{aligned}
$$

Substituting above expressions into Eq. (21) and taking a logarithm, we obtain the entropy of the black hole

$$
\begin{aligned}
S= & \ln \rho \approx 2 \pi \sqrt{\frac{c \Delta}{6}}+\frac{1}{4} \ln \frac{c}{\Delta^{3}}+\cdots \\
S= & \frac{A_{d-1}}{4 G_{D}}\left(1-\frac{2(D-2) \lambda L^{2}}{(D-4) r_{h}^{2}}\right) \\
& -\frac{3}{2} \ln \left[\frac{A_{d-1}}{4 G_{D}}\left(1-\frac{2(D-2) \lambda L^{2}}{(D-4) r_{h}^{2}}\right)\right]+\cdots \\
= & S_{\text {Wald }}-\frac{3}{2} \ln S_{\text {Wald }}+\cdots .
\end{aligned}
$$

In the above equation, the first term is exactly the Wald entropy of a Gauss-Bonnet black hole in $D$-dimensions. We also find the logarithmic correction to the Wald entropy with coefficient $-3 / 2$, which is the same as in the AdSSchwarzschild black hole case. As one can see, however, now in terms of horizon radius, there are two correction terms.

Before ending this section, it is worthwhile to point out again the debatable nature of the coefficient of logarithmic term in black hole entropy. The logarithmic correction to the usual black hole entropy has been discussed in various quantum gravity models. This correction term has been successfully computed for asymptotically flat black holes in loop quantum gravity (LQG), in string theory for both extremal and near extremal black holes by microscopic counting methods or by diffeomorphism symmetry arguments which heavily rely on two dimensional CFT and the Cardy formula (as 
we have done in this section). All of these methods have either predicted different coefficient for the logarithmic term or have a certain level of arbitrariness in their results. For example, in LQG the coefficient was found to be $-3 / 2$ [14]. However, in string theory, this coefficient was found to be different from $-3 / 2$ [15-18]. There, the logarithmic corrections to the entropy of the black hole arise from the one-loop contribution of the massless fields, and the coefficient was found to be dependent on the total number of massless fields as well as on the number of spacetime dimensions.

For the BTZ black hole, Carlip calculated this coefficient using the Cardy formula and found it to be $-3 / 2$ [35]. He also argued that similar logarithmic correction should appear in all black holes whose microscopic degrees of freedom are described by an underlying horizon CFT. However, in his calculation Carlip had assumed, as we have also assumed in this paper, that the central charge $c$ is universal constant in a sense that it is independent of the area of the horizon. To achieve this assumption Carlip chose the parameter $\alpha$ (appearing in Eq. (36)) in such a way that $c$ becomes independent of area. It is important to point out here that one could choose $\alpha$ to be the surface gravity $\kappa$ as well. The condition $\alpha=\kappa$ is also well motivated from Euclidean gravity point of view and is standard in the literature. ${ }^{8}$ To see this, let us consider the Euclidean time $(\tau \rightarrow i t)$ and take the appropriate ansatz for $T_{m}$ as $T_{m}=1 / \alpha e^{i m(\alpha \tau+g(\tilde{r})+\vec{p} \cdot \vec{x})}$. In the Euclidean formalism our analysis still go through, but now $\tau$ must have a periodicity of $2 \pi / \kappa$ to avoid the conical singularity. In order to maintain this periodicity in $\tau$, we must choose $\alpha=\kappa$. If we choose this condition then its not hard to see that the coefficient of the logarithmic correction is $-1 / 2$ instead of $-3 / 2$.

From above discussion, it is therefore fair to say that as of now there is no consensus on the coefficient of this logarithmic correction term and that more work in needed in order to say anything conclusively. Since we are mostly interested in studying the effects of this logarithmic correction term on the holographic Rényi entropy, we adopt here a more neutral point of view and consider a general logarithmic correction with arbitrary coefficient $\mathbf{C}$, instead of worrying too much about its exact magnitude

$S=S_{\text {Wald }}+\mathbf{C} \ln S_{\text {Wald }}+\cdots$.

The whole purpose of our previous exercise, where we computed $\mathbf{C}=-3 / 2$, was to show at-least one method by which logarithmic correction to the entropy of topological black holes, not just in Einstein gravity but in other higher derivative gravity too, can be explicitly calculated and that this

\footnotetext{
${ }^{8}$ In various methods of computing the entropy of the black hole from diffeomorphism symmetry arguments, the parameter $\alpha$ is generally chosen to be the surface gravity by hand to get the correct expression for the entropy of the black hole; see for instance $[31,44,50]$.
}

coefficient is non-zero. It would certainly be interesting to generalize the method of [14] in LQG or of [17] in string theory to calculate logarithmic correction for topological black holes. Especially, for higher derivative gravity theories it is not clear (at-least to the author) how to proceed. It would be an interesting problem in its own right to analyze similarities and differences in the results of these methods but it is beyond the scope of this paper.

Let us also point out that the horizon Virasoro algebra constructed in Eqs. (41) and (50) are not related to the boundary CFT and its corresponding algebra i.e. to the CFT that appears in AdS/CFT language. They are two different and separate things. It is important to keep this in mind. The horizon Virasoro algebra is constructed only to get the entropy of the black hole expression and after that it does not appear any where in the holographic Rènyi entropy calculation, as we will see in the next section.

\section{Calculations and results}

In this section, we will present results for the holographic Rényi entropy by considering logarithmic correction to the usual entropy of the black hole. We will again concentrate on two gravity theories: Einstein and Gauss-Bonnet. Our main focus here will be to see the effects of this logarithmic correction on the Rényi entropy. However, before going into the details of each case separately, let us first calculate the area of the hyperbolic event horizon appearing in Eqs. (44) and (53). The line element on the $(d-1)$-dimensional hyperbolic space is given in Eq. (38), which we reproduce here again for convenience

$\mathrm{d} \Sigma_{d-1}^{2}=\mathrm{d} \theta^{2}+\sinh ^{2} \theta \mathrm{d} \Omega_{d-2}^{2}$.

It would be convenient if we make a change of coordinate $\theta=\cosh ^{-1} y$, in which case the above metric reduces to

$\mathrm{d} \Sigma_{d-1}^{2}=\frac{\mathrm{d} y^{2}}{y^{2}-1}+\left(y^{2}-1\right) \mathrm{d} \Omega_{d-2}^{2}$.

The area of the hyperbolic space (and hence the area of the event horizon) is divergent. In order to regulate this area, we introduce a upper cutoff by integrating out to a maximum radius

$y_{\max }=\frac{R}{\delta}$,

where $\delta$ is the short distance cutoff related to the UV cutoff of the boundary CFT (see [8] for more details). Now, the area $V_{\Sigma_{d-1}}$ of the hyperbolic space is calculated as 


$$
\begin{aligned}
& V_{\Sigma_{d-1}}=\Omega_{d-2} \int_{1}^{y_{\max }} \mathrm{d} y\left(y^{2}-1\right)^{(d-3) / 2} \\
& \simeq \frac{\Omega_{d-2}}{d-2}\left[\left(y_{\max }^{d-2}-1\right)-\frac{(d-2)(d-3)}{2(d-4)}\left(y_{\max }^{d-4}-1\right)\right. \\
& \left.\quad+\frac{(d-2)(d-3)(d-5)}{8(d-6)}\left(y_{\max }^{d-6}-1\right)-\cdots\right] \\
& \simeq \frac{\Omega_{d-2}}{d-2} \\
& \quad \times\left[\frac{R^{d-2}}{\delta^{d-2}}-\frac{(d-2)(d-3)}{2(d-4)} \frac{R^{d-4}}{\delta^{d-4}}\right. \\
& \left.\quad+\frac{(d-2)(d-3)(d-5)}{8(d-6)} \frac{R^{d-6}}{\delta^{d-6}}-\cdots\right]
\end{aligned}
$$

where $\Omega_{d-2}=2 \pi^{(d-1) / 2} / \Gamma((d-1) / 2)$ is the area of a unit $(d-2)$ sphere. However, for $d=2$, we have a logarithmic behavior

$$
\begin{aligned}
V_{\Sigma_{1}} & =2 \int_{1}^{y_{\max }} \mathrm{d} y\left(y^{2}-1\right)^{-1 / 2}=2 \ln \left(2 y_{\max }\right) \\
& =2 \ln \left(\frac{2 R}{\delta}\right) .
\end{aligned}
$$

Therefore, the area of the event horizon $A_{d-1}$ is given by

$A_{d-1}=r_{h}^{d-1} V_{\Sigma_{d-1}}$.

Now we have all the ingredients to compute the holographic Rényi entropy for spherical entangling surface.

\subsection{Rényi entropy from AdS-Schwarzschild black hole}

For AdS-Schwarzschild Black hole the metric is given in Eq. (37). However, in order to make comparison with [10], let us rewrite this in a slightly different form

$$
\begin{aligned}
\mathrm{d} s^{2}= & -\left[-1+\frac{r^{2}}{L^{2}} g(r)\right] N^{2} \mathrm{~d} t^{2}+\frac{1}{\left[-1+\frac{r^{2}}{L^{2}} g(r)\right]} \mathrm{d} r^{2} \\
& +r^{2} \mathrm{~d} \Sigma_{d-1}^{2}, \\
g(r)= & \frac{L^{2}}{r^{2}}(f(r)+1)
\end{aligned}
$$

In order to ensure that the boundary spacetime is conformally equivalent to $R \times H^{d-1}$, i.e.

$\mathrm{d} s_{\infty}^{2}=-\mathrm{d} t^{2}+R^{2} \mathrm{~d} \Sigma_{d-1}^{2}$

we choose the constant $N^{2}=L^{2} /\left(g_{\infty} R^{2}\right)=\tilde{L}^{2} / R^{2}$, where $g_{\infty}=\lim _{r \rightarrow \infty} g=1$. The Hawking temperature of this black hole is given by

$T=\frac{1}{4 \pi R}\left(\frac{\mathrm{d} r_{h}}{L}-\frac{(d-2) L}{r_{h}}\right)$,

which is also the temperature of the boundary CFT on $R \times H^{d-1}$. For computational purposes, it is convenient to consider a coordinate $x=r_{h} / \tilde{L}$ in which case the expression for the Rényi entropy in Eq. (11) reduces to

$$
\begin{aligned}
S_{q} & =\frac{q}{q-1} \frac{1}{T_{0}} \int_{x_{q}}^{1} \mathrm{~d} x S_{\text {therm }}(x) \frac{\mathrm{d} T}{\mathrm{~d} x} \\
& =\frac{q}{q-1} \frac{1}{T_{0}}\left[\left.S_{\text {therm }}(x) T(x)\right|_{x_{q}} ^{1}-\int_{x_{q}}^{1} \mathrm{~d} x T(x) \frac{\mathrm{d}}{\mathrm{d} x} S_{\text {therm }}\right] .
\end{aligned}
$$

Here, in the above integral, the upper limit $x=1$ comes from the condition $T=T_{0}$, which implies $r_{h}=\tilde{L}$. The lower limit $x_{q}$, which needs to be determined, corresponds to the temperature $T=T_{0} / q$. Using Eq. (63), $x_{q}$ must satisfy the following equation:

$\mathrm{d} x_{q}^{2}-\frac{2}{q} x_{q}-(d-2)=0$

the real and positive root of which is given by

$x_{q}=\frac{1}{q d}\left[1+\sqrt{d^{2} q^{2}+1-2 \mathrm{~d} q^{2}}\right]$.

Finally, using the expression for the entropy of the black hole (Eq. (54)) in place of $S_{\text {therm }}$ in Eq. (64), we get the expression for Rényi entropy as

$S_{q}=\frac{q \mathfrak{B}}{2(q-1)}\left(2-x_{q}^{d-2}\left(1+x_{q}^{2}\right)\right)+\frac{\mathbf{C} q}{q-1} \ln \mathfrak{B}+\frac{\mathbf{C} q}{2(q-1) x_{q}}$ $\times\left[(d-1)\left(x_{q}-1\right)\left(\mathrm{d} x_{q}+2-d\right)-\left(\mathrm{d} x_{q}^{2}+2-d\right) \ln \left(\mathfrak{B} x_{q}^{d-1}\right)\right]$,

where $\mathfrak{B}=2 \pi V_{\Sigma_{d-1}}\left(\frac{\tilde{L}}{l_{p}}\right)^{d-1}, l_{p}$ is the Planck length related to the gravitation constant $G_{D}=l_{p}^{d-1} / 8 \pi$. We see that $S_{q}$ is a complicated function of $x_{q}$. For $\mathbf{C}=0, S_{q}$ reduces to the expression found in [10]. However, for $\mathbf{C} \neq 0$ there are additional non-trivial correction terms. We now make some observations:

- For $d=2$, we get

$$
\begin{aligned}
& S_{q}(d=2)=\frac{\mathfrak{B}}{2}\left(1+\frac{1}{q}\right)+\mathbf{C}(\ln (\mathfrak{B})-1)+\mathbf{C} \frac{\ln q}{q-1} \\
& =\frac{c}{6}\left(1+\frac{1}{q}\right) \ln \left(\frac{2 R}{\delta}\right)+\mathbf{C}\left[\ln \left(\frac{c}{3} \ln \left(\frac{2 R}{\delta}\right)\right)-1\right] \\
& \quad+\mathbf{C} \frac{\ln q}{q-1},
\end{aligned}
$$

where $c=12 \pi L / l_{p}$ is the standard expression of the central charge in the two dimensional boundary CFT. ${ }^{9}$ The first term in Eq. (68) matches with the well known result of the

\footnotetext{
9 This $c$ should not be confused with the central charge of Eq. (43) which appears in the Virasoro algebra on the horizon. The $c$ in Eq. (43) was used to compute the black hole entropy and has nothing to do with the boundary CFT.
} 
Rényi entropy in a two dimensional CFT for an interval of length $l=2 R$. This is expected as, for $d=2$, the spherical entangling region consists of two points separated by distance $2 R$. However, our main result here is the appearance of additional corrections to Rényi entropy which are precisely coming from the coefficient of the logarithmic correction to the entropy of the black hole. These correction terms to the Rényi entropy have both additional logarithmic as well as double logarithmic structure.

- Similarly, the entanglement entropy for $d=2$ is obtained by taking $q \rightarrow 1$ limit of Eq. (68)

$$
S_{1}(d=2)=\frac{c}{3} \ln \left(\frac{2 R}{\delta}\right)+\mathbf{C}\left[\ln \left(\frac{c}{3} \ln \left(\frac{2 R}{\delta}\right)\right)\right]
$$

The leading term again has the same structure as is well known for the two dimensional CFT. However, now we have found additional correction too. Interestingly, the correction term in the entanglement entropy has a simple structure. It is proportional to $\mathbf{C}$ times the logarithm of its standard expression. Moreover, the same is true in all dimensions. That is,

$$
\begin{aligned}
S_{1}(d)= & \mathfrak{B}+\mathbf{C} \ln [\mathfrak{B}] \\
= & 2 \pi V_{\Sigma_{d-1}}\left(\frac{\tilde{L}}{l_{p}}\right)^{d-1}+\mathbf{C} \ln \left[2 \pi V_{\Sigma_{d-1}}\left(\frac{\tilde{L}}{l_{p}}\right)^{d-1}\right] \\
= & 2 \pi V_{\Sigma_{d-1}}\left(\frac{\tilde{L}}{l_{p}}\right)^{d-1}+\mathbf{C} \ln \left[\left(\frac{\tilde{L}}{l_{p}}\right)^{d-1}\right] \\
& +\mathbf{C} \ln \left[2 \pi V_{\Sigma_{d-1}}\right] .
\end{aligned}
$$

In the last line, we have divided the correction term into two parts. One that depends on $\left(\tilde{L} / l_{p}\right)^{d-1}$, which is related to the number of degrees of freedom of the boundary CFT and the other that depends on the size of the system. Let us concentrate on the term which is of order $G_{D}^{0}$ (last term in Eq. (70)). ${ }^{10} \mathrm{We}$ observe that at this order there is a logarithmic correction in all dimensions. In particular, there is a logarithmic correction in odd $d$ as well. In order to see this, we write the complete expression for entanglement entropy in $d=3$ and 4 dimensions

$$
\begin{aligned}
S_{1}(d=3)= & \left(2 \pi \frac{\tilde{L}}{l_{p}}\right)^{2}\left(\frac{R}{\delta}-1\right) \\
& +\mathbf{C} \ln \left(\frac{\tilde{L}}{l_{p}}\right)^{2}+\mathbf{C} \ln \left(4 \pi^{2} \frac{R}{\delta}\right)+\cdots
\end{aligned}
$$

\footnotetext{
${ }^{10}$ Quantum corrections to the RT formula are expected to occur at the order $G_{D}^{0}[40]$. The RT formula gives entanglement entropy at the order $1 / G_{D}$. See the discussion section for more details.
}

$$
\begin{aligned}
S_{1}(d=4)= & 4 \pi^{2}\left(\frac{\tilde{L}}{l_{p}}\right)^{3}\left(\frac{R^{2}}{\delta^{2}}-\ln \frac{2 R}{\delta}\right) \\
& +\mathbf{C} \ln \left(\frac{\tilde{L}}{l_{p}}\right)^{3}+\mathbf{C} \ln \left(4 \pi^{2} \frac{R^{2}}{\delta^{2}}\right)+\cdots,
\end{aligned}
$$

we note from the last terms of Eq. (71) that at the order $G_{D}^{0}$ there is a correction to the entanglement entropy which depends logarithmically on the size of the entangling surface. The same result holds in all $d>2$ dimensions.

- At first sight the results in Eq. (69)-(71) look strange as they predict additional logarithmic divergence in the entanglement entropy and seems to violate $c$-theorem like results. However, as we will show shortly this pessimism is unjustified.

As is well known, the entanglement entropy suffers from UV divergences, and as a result it is ill-defined in the continuum limit i.e. $\delta \rightarrow 0$. A normal practice is to subtract the UV divergent part (in an ad hoc way) by hand, a practice which is ambiguous and often not unique. In [37], a procedure for "renormalized entanglement entropy" was given which is intrinsically UV finite and predicts well defined properties of the entanglement entropy. In particular, depending on spacetime dimensions, following renormalized expressions of the entanglement entropy $\left(\mathcal{S}_{1}(R)\right)$ were proposed,

$$
\begin{aligned}
\mathcal{S}_{1}(R)= & \frac{1}{(d-2) ! !}\left(R \frac{\mathrm{d}}{\mathrm{d} R}-1\right)\left(R \frac{\mathrm{d}}{\mathrm{d} R}-3\right) \\
& \ldots\left(R \frac{\mathrm{d}}{\mathrm{d} R}-(d-2)\right) S_{1}, \text { for odd } \mathrm{d}, \\
= & \frac{1}{(d-2) ! !} R \frac{\mathrm{d}}{\mathrm{d} R}\left(R \frac{\mathrm{d}}{\mathrm{d} R}-2\right) \\
& \ldots\left(R \frac{\mathrm{d}}{\mathrm{d} R}-(d-2)\right) S_{1}, \text { for even } \mathrm{d} .
\end{aligned}
$$

In [37], It was shown that (i) the renormalized entanglement entropy $\mathcal{S}_{1}(R)$ is UV finite in the continuum limit, (ii) it is a monotonic non-negative function that decrease form UV to IR, therefore satisfying the $c$-theorem like functions, and (iii) it may be considered as the "universal part" of the original entanglement entropy, a part which can be defined intrinsically in the continuum limit. We can clearly see that all these properties are explicitly satisfied in the corrected entanglement entropy expression as well. In particular, for $d=2$ we have

$\mathcal{S}_{1}(d=2)=R \frac{\mathrm{d}}{\mathrm{d} R} S_{1}=\frac{c}{3}+\frac{\mathbf{C}}{\ln (2 R / \delta)}$,

$\left.\mathcal{S}_{1}(d=2)\right|_{\delta=0}=\frac{c}{3}$

therefore, $\mathcal{S}_{1}(d=2)$ is again UV finite in the continuum limit. Moreover, the finite/and or universal part of the renormalized entanglement entropy remains the same even by taking the corrections to the original entanglement entropy 
expression. Since $c$ is the central charge of two dimensional CFT which generally decreases from UV to IR i.e. $c^{(U V)}>c^{I R}$, it indicates that the renormalized entanglement entropy is also a monotonic non-negative function that decrease form UV to IR. ${ }^{11}$ Accordingly, the $c$-theorem is again satisfied. Therefore, the additional logarithmic divergences in the entanglement entropy do not lead to any nontrivial issues as far as the entropic $c$-function and universality is concerned. Moreover, one can also explicitly check that in higher dimensions as well the renormalized entanglement entropy is again UV finite and is non-negative monotonic function. In Sect. 6, we will give another reason, based on the results of one-loop test of AdS/CFT, why logarithmic divergences in the entanglement entropy are expected especially in odd dimensions.

- For $d=2$, the ratio of Rényi entropy to entanglement entropy as a function of $q$ for some reasonable values of $\mathbf{C}$ is plotted in Fig. 1. In order to plot this ratio, we have chosen $R=1, \delta=10^{-4}$ and $L=2 l_{p}$. We see that the overall behavior of $S_{q} / S_{1}$ is the same for all $\mathbf{C}$. For a fixed $q>1$, the magnitude of the ratio $S_{q} / S_{1}$ decreases with decrease in $\mathbf{C}$. However, we should emphasize here that these results are cutoff $(\delta)$ dependent. For instance, for much smaller cutoff, say $\delta=10^{-20}$, differences due to $\mathbf{C} \neq 0$ are almost negligible. Similarly, we also found that, for larger and larger values of $c$ ( or $L / l_{p}$ ), the effect of $\mathbf{C}$ become smaller and smaller. We can also note that $S_{q} / S_{1}>1$ for $q<1$ and $S_{q} / S_{1}<1$ for $q>1$.

- Let us also note some useful limits of the Rényi entropy

$$
\begin{aligned}
S_{\infty}= & \mathfrak{B}\left[1-\frac{d-1}{d}\left(\frac{d-2}{d}\right)^{\frac{d-2}{2}}\right] \\
& +\mathbf{C}\left[\ln \mathfrak{B}-(d-1)^{2}+(d-1) \sqrt{d(d-2)}\right] \\
S_{1}= & \mathfrak{B}+\mathbf{C} \ln \mathfrak{B} \\
S_{0}= & \mathfrak{B}\left[\left(\frac{2}{d}\right)^{d} \frac{1}{2 q^{d-1}}+\left(\frac{2}{d}\right)^{d-2} \frac{1}{2 q^{d-3}}\right] \\
& +\mathbf{C}[\ln \mathfrak{B}-(d-1)-(d-1) \ln (\mathrm{d} q)] .
\end{aligned}
$$

- Now, we discuss Rényi and entanglement entropies for higher dimensional theories. In Fig. 2, $S_{q} / S_{1}$ as a function of $q$ for various values of $d$ is shown. Here, we have considered $\mathbf{C}=-3 / 2$ and again have chosen $R=1, \delta=10^{-4}$ and $L=$ $2 l_{p}$. The overall characteristic features of $S_{q} / S_{1}$ is found to be same as in the $d=2$ case. We found that, for fixed $\mathbf{C}$, as we

\footnotetext{
11 For $d=2$, the expression for renormalized entanglement entropy is similar to the entropic $c$-function [51]. The fact that $c$-function decreases monotonically from UV to IR has also been confirmed both in lattice as well as in holographic studies [52,53].
}

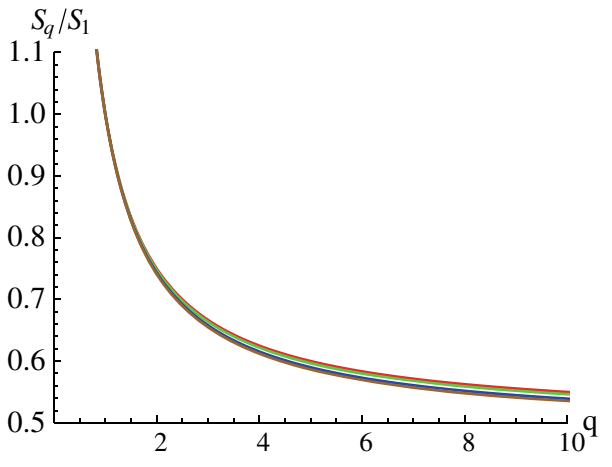

Fig. $1 S_{q} / S_{1}$ as a function of $q$ for various C. Here $d=2$ and red, green, blue and brown curves correspond to $\mathbf{C}=0,-1 / 2,-3 / 2$ and -2 , respectively

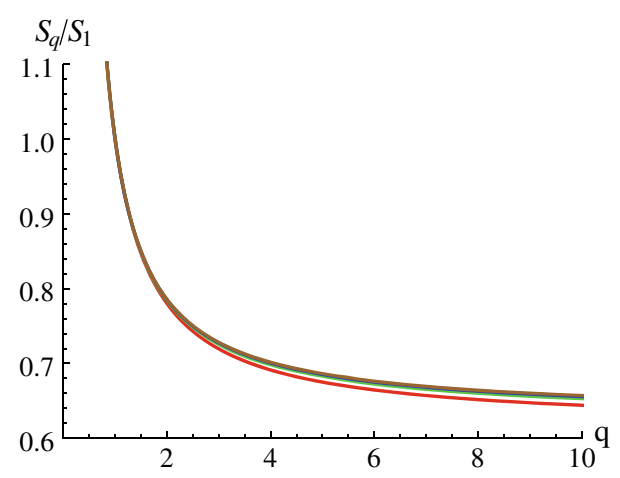

Fig. $2 S_{q} / S_{1}$ as a function of $q$ for various $d$. Here $\mathbf{C}=-3 / 2$ and red, green, blue and brown curves correspond to $d=3,4,5$ and 6 , respectively

increase the number of spacetime dimensions the magnitude of the ratio $S_{q} / S_{1}$ increases. Even for large $d$, we again found that $S_{q} / S_{1}>1$ for $q<1$ and $S_{q} / S_{1}<1$ for $q>1$.

- Let us also comment on some of the useful inequalities of the Rényi entropy. It is well known that Rényi entropy satisfies inequalities involving the derivative with respect $q$, such as

$$
\begin{aligned}
\partial_{q} S_{q} & \leq 0 \\
\partial_{q}\left[\frac{q-1}{q} S_{q}\right] & \geq 0 \\
\partial_{q}\left[(q-1) S_{q}\right] & \geq 0 \\
\partial_{q}^{2}\left[(q-1) S_{q}\right] & \leq 0 ;
\end{aligned}
$$

therefore, it is natural to ask whether or not these inequalities are satisfied with the corrected results. For $d>2$, we find that these inequalities are indeed satisfied provided $\mathbf{C}$ is not very large (which is expected for small corrections). However, for $d=2$, we find few subtleties, especially in the fourth inequality of Eq. (75). We find that for small central charge it can be violated. Although for large central charge, which 
is the case for boundary theories that have a gravity dual, the fourth inequality is again found to be satisfied.

- Perhaps, the most important result from our computation is that the leading UV divergences appearing in Eq. (67) is same for all $q$. Indeed, if we note that leading term $R^{d-2} \Omega_{d-2}$ in Eq. (58) gives the area of the spherical entangling region, then the leading divergent term always has a "area law" structure for $d>2$. For $d=2$, we have the usual logarithmic divergence in the Rényi entropy. As expected, the correction terms do not change the leading behavior of the Rényi entropy.

\subsection{Rényi entropy from Gauss-Bonnet black hole}

In this subsection, we examine the holographic Rényi entropy for boundary theory which are dual to Gauss-Bonnet gravity theory. The procedure for calculating Rényi entropy with Gauss-Bonnet Black hole background is entirely similar to what has been discussed in the previous subsection, and therefore we will be brief here.

The metric for Gauss-Bonnet Black hole is given in Eq. (47), however, we rewrite it in a slightly different form in order to make comparison with [10]

$$
\begin{aligned}
\mathrm{d} s^{2}=- & {\left[-1+\frac{r^{2}}{L^{2}} g(r)\right] N^{2} \mathrm{~d} t^{2} } \\
& +\frac{1}{\left[-1+\frac{r^{2}}{L^{2}} g(r)\right]} \mathrm{d} r^{2}+r^{2} \mathrm{~d} \Sigma_{d-1}^{2}, \\
g(r)= & \frac{1}{2 \lambda}\left[1-\sqrt{1-4 \lambda+\frac{4 \lambda m}{r^{d}}}\right] .
\end{aligned}
$$

In this case $N^{2}=L^{2} /\left(g_{\infty} R^{2}\right)=\tilde{L}^{2} / R^{2}$. Since now $g_{\infty}=$ $(1-\sqrt{1-4 \lambda}) /(2 \lambda) \neq 1$, it implies $\tilde{L} \neq L$. This is just the statement that the AdS curvature scale is distinct from the length scale $L$, which usually appears in the Gauss-Bonnet gravity action. Now, again using the coordinate $x=r_{h} / \tilde{L}$, the Hawking temperature can be expressed as

$$
T=\frac{1}{2 \pi R x}\left(1+\frac{d}{2 g_{\infty}} \frac{x^{4}-x^{2} g_{\infty}+\lambda g_{\infty}^{2}}{x^{2}-2 \lambda g_{\infty}}\right) .
$$

Similarly, the horizon entropy (Eq. (53)) can be recast as

$$
\begin{aligned}
S= & 2 \pi\left(\frac{\tilde{L}}{l_{p}}\right)^{d-1} V_{\Sigma_{d-1}} x^{d-1}\left(1-\frac{2 \lambda g_{\infty}}{x^{2}} \frac{d-1}{d-3}\right) \\
& +\mathbf{C} \ln \left[2 \pi\left(\frac{\tilde{L}}{l_{p}}\right)^{d-1} V_{\Sigma_{d-1}} x^{d-1}\right. \\
& \left.\times\left(1-\frac{2 \lambda g_{\infty}}{x^{2}} \frac{d-1}{d-3}\right)\right] .
\end{aligned}
$$

After substituting above results into Eq. (64), we can get expression for the Rényi entropy for any $d$. However, for general $d$ the expressions for Rényi entropy is very complicated and lengthy and not very illuminating. For this reason we focus on $d=4$ case, in which the Rényi entropy obeys the following expression:

$$
\begin{aligned}
S_{q}= & \frac{q \mathfrak{B}}{2(q-1)} \\
& \times\left[\frac{1-x_{q}^{3}}{g_{\infty}}-3\left(1-x_{q}^{2}\right)+4(1-4 \lambda)\right. \\
& \left.\times\left(\frac{1}{1-2 \lambda g_{\infty}}-\frac{x_{q}^{4}}{x_{q}^{2}-2 \lambda g_{\infty}}\right)\right]+ \\
& \times \frac{\mathbf{C} q\left(g_{\infty}-2\right)}{(q-1) g_{\infty}\left(2 \lambda g_{\infty}-1\right)} \ln \left[\mathfrak{B}\left(1-6 \lambda g_{\infty}\right)\right] \\
& -\frac{\mathbf{C} q x_{q}\left(g_{\infty}-2 x_{q}^{2}\right)}{(q-1) g_{\infty}\left(2 \lambda g_{\infty}-x_{q}^{2}\right)} \ln \left[\mathfrak{B} x_{q}\left(x_{q}^{2}-6 \lambda g_{\infty}\right)\right] \\
& +\frac{6 \mathbf{C} q}{(q-1)} \frac{\left(x_{q}-1\right)}{g_{\infty}}+\frac{\sqrt{3} \mathbf{C} q(12 \lambda-1)}{(q-1) \sqrt{2 \lambda g_{\infty}}} \\
& \times\left[\tanh ^{-1}\left(\frac{1}{\sqrt{6 \lambda g_{\infty}}}\right)-\tanh { }^{-1}\left(\frac{x_{q}}{\sqrt{6 \lambda g_{\infty}}}\right)\right],
\end{aligned}
$$

where $x_{q}$ corresponds to the positive and real root of the following equation:

$4 q x_{q}^{4}-2 g_{\infty} x_{q}^{3}-2 q g_{\infty} x_{q}^{2}+4 \lambda g_{\infty}^{2} x_{q}=0$,

which is again obtained from the equation $T=T_{0} / q$, i.e. for the lower limit of the integral in Eq. (64). $\lambda$, which appears in the argument of logarithmic and inverse hyperbolic functions in Eq. (79) should be understood as an absolute value. The first term in Eq. (79) have the same expression for Rényi entropy as was found in [10]. However, now we also have additional correction terms, which are both logarithmic as well as non-logarithmic in nature. One can also explicitly check that, in the limit $\lambda \rightarrow 0$, Eq. (79) reduces to Eq. (67) for Einstein gravity.

We now make some observations:

- It is well known that the dual four dimensional boundary CFT of five dimensional Gauss-Bonnet gravity theory has two distinct central charges ${ }^{12}$

$$
c=\pi^{2}\left(\frac{\tilde{L}}{l_{p}}\right)^{3}\left(1-2 \lambda g_{\infty}\right), \quad a=\pi^{2}\left(\frac{\tilde{L}}{l_{p}}\right)^{3}\left(1-6 \lambda g_{\infty}\right) .
$$

In terms of these central charges the Rényi entropy in Eq. (79) reduces to

$$
\begin{aligned}
& S_{q}=\frac{V_{\Sigma_{3}}}{4 \pi} \frac{q\left(1-x_{q}^{2}\right)}{q-1} \\
& \times\left[(5 c-a) x_{q}^{2}-(13 c-5 a)+16 c \frac{2 c x_{q}^{2}-(c-a)}{(3 c-a) x_{q}^{2}-(c-a)}\right]
\end{aligned}
$$

\footnotetext{
12 Again, these central charges should not be confused with the central charge of Eq. (43).
} 


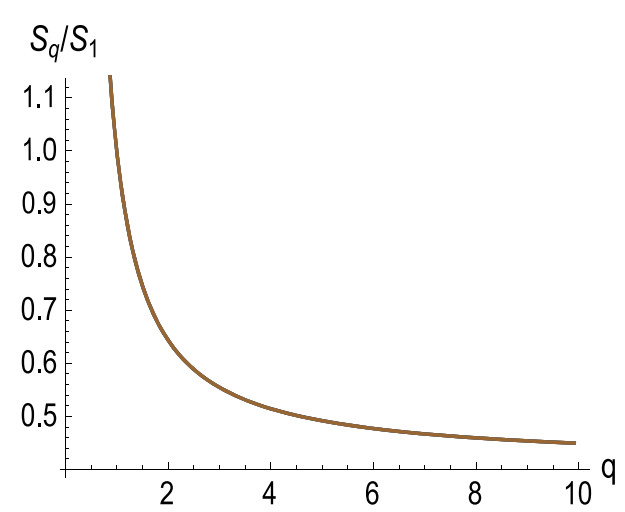

Fig. $3 S_{q} / S_{1}$ as a function of $q$ for various C. Here $\lambda=0.08$ and red, green, blue and brown curves correspond to $\mathbf{C}=0,-1 / 2,-3 / 2$ and -2 respectively. For this plot, we have chosen $R=1, \delta=10^{-4}$ and $L=2 l_{p}$

$$
\begin{aligned}
& +\frac{\mathbf{C} q}{(q-1)} \ln \left[\frac{2 a V_{\Sigma_{3}}}{\pi}\right]-\frac{\mathbf{C} q x_{q}}{(q-1)} \frac{\left((5 c-a) x_{q}^{2}-3 c+a\right)}{\left((3 c-a) x_{q}^{2}-c+a\right)} \\
& \times \ln \left[\frac{V_{\Sigma_{3}} x_{q}}{\pi}\left((3 c-a) x_{q}^{2}-3 c+3 a\right)\right] \\
& +\frac{2 \mathbf{C} q}{(q-1)} \frac{\left(3 c^{2}+a^{2}-6 a c\right)}{(3 c-a)^{2}} \sqrt{\frac{3(3 c-a)}{c-a}}\left[\tanh ^{-1} \sqrt{\frac{3 c-a}{3(c-a)}}\right. \\
& \left.-\tanh ^{-1} \sqrt{\frac{3 c-a}{3(c-a)} x_{q}^{2}}\right] \\
& +\frac{\mathbf{C} q\left(x_{q}-1\right)}{(q-1)} \frac{(15 c-3 a)}{(3 c-a)}
\end{aligned}
$$

which shows that the Rényi entropy is quite a complicated function of these central charges. It is also clear that the Rényi entropy is not determined solely by the anomaly coefficient $a$ as in the case of entanglement entropy (see below). As in the case of Einstein gravity, here too, the size of the entangling surface always appears logarithmic in the correction terms. This feature therefore seems to be universal in nature.

- We get the expression for the entanglement entropy as

$$
S_{1}=a \frac{2 V_{\Sigma_{3}}}{\pi}+\mathbf{C} \ln \left[a \frac{2 V_{\Sigma_{3}}}{\pi}\right]
$$

we see that, as in the Einstein gravity case, correction term to the entanglement entropy is still given by the logarithmic of its original expression. This result can be traced back to Eq. (11), where it is clear that entanglement entropy is nothing but the entropy of the black hole at temperature $T=T_{0}$. Therefore, a logarithmic correction to the entropy of the black hole implies logarithmic correction to the entanglement entropy. It is also easy to see that similar results hold in higher dimensions too.

- $S_{q} / S_{1}$ as a function of $q$ for some reasonable values of $\mathbf{C}$ and for $\lambda=0.08$ is shown in Fig. 3. We see that the

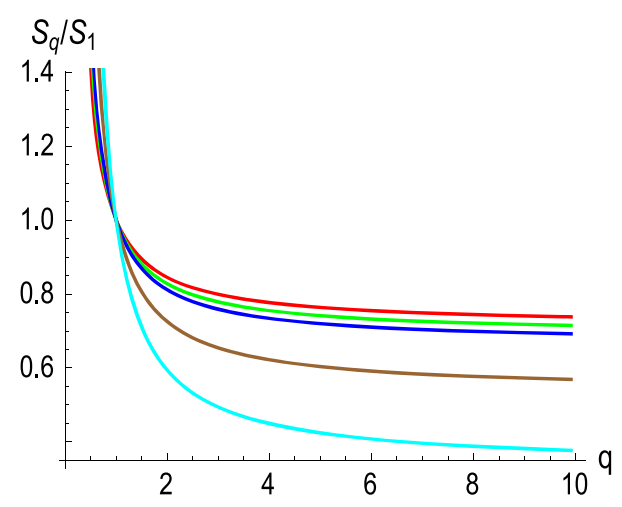

Fig. $4 S_{q} / S_{1}$ as a function of $q$ for various $\lambda$. Here $\mathbf{C}=-3 / 2$ and red, green, blue, brown and cyan curves correspond to $\lambda=-0.19,-0.1$, $-0.05,0.05$ and 0.09 , respectively. For this plot, we have chosen $R=1$, $\delta=10^{-4}$ and $L=2 l_{p}$

difference due to $\mathbf{C}$ is extremely small and that the overall behavior of $S_{q} / S_{1}$ is the same for all C. Similarly, $S_{q} / S_{1}$ for different values of $\lambda$ and for fixed $\mathbf{C}=-3 / 2$ is shown in Fig. 4. We find that $S_{q} / S_{1}$, in the region $q<1$, increases for higher and higher values of $\lambda$, however, in the region $q>1$, it decreases. Similar results hold for other values of $\mathbf{C}$.

- Before ending this section, let us also note some other useful limits of the Rényi entropy,

$$
\begin{aligned}
S_{0}= & \frac{V_{\Sigma_{3}}}{4 \pi} \frac{(3 c-a)^{4}}{(5 c-a)^{3}}\left(\frac{1}{q^{3}}+\frac{1}{q^{2}}\right) \\
& +\frac{V_{\Sigma_{3}}}{4 \pi q} \frac{(3 c-a)\left(5 a^{3}-57 a^{2} c+159 a c^{2}-3 c^{3}\right)}{(5 c-a)^{3}} \\
& -3 \mathbf{C} \ln q+\mathfrak{h}\left(c, a, V_{\Sigma_{3}}\right), \\
S_{\infty}= & \frac{V_{\Sigma_{3}}}{\pi} \frac{\left(10 a c-3 c^{2}-2 a^{2}\right)}{(5 c-a)}+\mathbf{C} \ln \left(2 a \frac{V_{\Sigma_{3}}}{\pi}\right) \\
& +\frac{3 \mathbf{C}(5 c-a)}{(3 c-a)}\left(\sqrt{\frac{3 c-a}{5 c-a}}-1\right) \\
& +\frac{2 \mathbf{C}\left(3 c^{2}+a^{2}-6 a c\right)}{(3 c-a)^{2} \sqrt{\frac{3(3 c-a)}{c-a}}}\left[\tanh ^{-1} \sqrt{\frac{(3 c-a)}{3(c-a)}}\right. \\
& \left.-\operatorname{coth}^{-1} \sqrt{3-\frac{12 c^{2}}{(3 c-a)^{2}}}\right]
\end{aligned}
$$

where $\mathfrak{h}$ is some function of $c, a$ and $V_{\Sigma_{3}}$ but independent of $q$. The leading diverging term of $S_{0}$ matches exactly with [10].

\section{Discussions and conclusions}

In this paper, we have studied the effects of the logarithmic correction of the entropy of the black hole on the Rényi entropy of a spherical entangling surface. We first used the 
diffeomorphism symmetry argument at the horizon to compute the black hole entropy expression. We used the Noether currents associated with the diffeomorphism invariance of the Gibbons-Hawking boundary action to construct the Virasoro algebra at the hyperbolic event horizons and then used this algebra to calculate the entropy of AdS-Schwarzschild and Gauss-Bonnet black holes. We found that the leading term in the expression for the entropy of the black hole is the usual Wald entropy and that there is a correction to it. This correction was found to be proportional to the logarithm of the area of the horizon.

We then applied the prescription of $[8,10]$ to calculate the holographic Rényi entropy for a spherical entangling surface. Using the corrected entropy of the black hole expression we found that there are corrections to the standard expression of Rényi entropy. These corrections are shown in Eq. (67) for Einstein and in Eq. (82) for Gauss-Bonnet gravity duals. In particular, we found that the Rényi entropy is a complicated function of the index $q$ and the central charges. Interestingly, the size of the entangling surface always appears logarithmically in the correction terms of the Rényi entropy. This is true for both Einstein and Gauss-Bonnet gravity. We found that the inequalities of the Rényi entropy as well as the entropic $c$-functions are satisfied even with the correction terms.

It is important to analyze the nature and significance of these correction terms in the Rényi entropy. If the corrections in the Rényi entropy originating from the corrections in black hole entropy are quantum corrections, then our results can be useful in many directions. Especially since a lot of work have recently been appeared in the literature to compute leading quantum corrections ( of order $G_{D}^{0}$ or $N^{0}$ ) to the entanglement entropy holographically. In this context, a proposal for quantum corrections to holographic entanglement entropy is given in [40], also see [54]. In this proposal, the quantum corrections are essentially given by the bulk entanglement entropy between RT minimal-area surface and the rest of the bulk (see Figure (1) of [40]). As an example, the quantum correction to the entanglement entropy of the Klebanov-Strassler model in the large $N$ limit was calculated. The correction was found to be, as in our case, logarithmic in nature and it depends on the size of the entangling surface. Indeed, one can also notice from Eq. (70) that at the order $G_{D}^{0}$ (recall that the factor $\left(\tilde{L} / l_{p}\right)^{d-1}$ measures the number of degrees of freedom of the dual CFT and it is related to the central charge of the boundary CFT or to the $G_{D}$ on the gravity side) the correction to the entanglement entropy is proportional to the logarithm of the size of the entangling surface. If the above interpretation is correct, then our results can be useful since they predict a similar kind of logarithmic correction to the Rényi entropy.

An indirect hint for the logarithmic correction in the Rényi entropy can also be found in the following way. In [18], a non-trivial test of the gauge/gravity duality at next-to-leading order in the $1 / N$ expansion for ABJM theories was performed. There, it was shown that the subleading logarithmic correction in the partition function (more correctly in $\log Z$ ) of the ABJM theory on a three sphere matches exactly with the partition function of its 11-dimensional supergravity dual. The latter partition function at one-loop level was calculated using the Euclidean quantum gravity method. Now, the subleading logarithmic term in the partition function can lead to a logarithmic correction in the Rényi entropy, provided that an analogous logarithmic term in the partition function on $q$-folded cover does not cancel in the definition of the Rényi entropy. This scenario is partially true, at least in odd dimensions.

Indeed one can notice, an important result of our analysis is that there are logarithmic corrections to entanglement and Rényi entropies in odd dimensions too. At first sight this seems strange as a logarithmic term in these entropies generally appears in even dimensions. However, it is also well known that the entanglement entropy for the sphere in flat space in odd dimensions is simply the negative of the free energy on a sphere $[56,57]$ i.e.,

$S_{1}=\log Z=-F_{1}$

it implies that any finite or divergent corrections in the partition function will lead to the same corrections in the entanglement entropy. Now since logarithmic corrections to the partition function on the sphere do appear at one-loop level, see for example [18], one can expect the same logarithmic corrections to appear in the entanglement entropy too. Therefore, it is possible to have a logarithmic term in the entanglement entropy in odd dimensions spacetime. However, this logarithmic term in the entanglement entropy arises only at one-loop level as compared to the logarithmic term in even spacetime dimensions which arises at tree level.

Since a similar kind of logarithmic subleading term in the partition function also arises in other boundary field theories, see for example [55], therefore it appears that logarithmic correction to the Rényi entropy might be a general feature of CFTs with gravity duals. Although, in order to explicitly establish this fact it would be useful if we can calculate the Rényi entropy at one-loop level on the lines of [41]. ${ }^{13}$ It would certainly be interesting to explicitly compare the results of our calculations with those of $[40,41]$, and find the similarities and differences between them.

Finally, regardless of the problems associated with the interpretation of correction terms calculated in this paper, it is important to carefully examine and explore their physical

\footnotetext{
${ }^{13}$ In [41], one-loop bulk corrections to RT formula was systematically calculated. This was done by calculating the one-loop determinants around the classical solutions using the Schottky uniformization of $q$ sheeted Riemann surface. However, there are many difficulties in implementing this method, such as constructing the smooth bulk solutions and performing analytic continuation of the replica index to non-integer $q$.
} 
significance. Especially since in the context of AdS/CFT, it is well known that on the gravity side the logarithmic correction to black hole entropy arises only due to the one-loop contribution of the massless fields. The fact that there are logarithmic corrections to the black hole entropy has been firmly established not just in string theory but also in loop quantum gravity. In [17], it was further pointed out by Sen that the logarithmic corrections to the entropy of the black hole are also universal in that they depend only on the massless spectrum of particles and are insensitive to the UV completion of the quantum gravity theory. Sen suggested that any microscopic theory of gravity will have a log correction to its entropy. Once we assume a logarithm correction to the entropy of the black hole as a well established fact, then this correction must have some correspondence to the holographic Rényi entropy via the mapping of $[8,10]$. What is this correspondence and how is the structure of the holographic Rényi entropy affected by this? This is a natural and important question to ask. In this paper we are suggesting that the logarithmic correction to the entropy of the black hole (if it exists) naturally leads to the logarithmic correction to holographic Rényi entropy. We believe this mapping can be further useful, not just to better understand the structure of the holographic Rényi entropy but also to get a better understanding of the coefficient of the logarithmic correction in the gravity side. We hope to comment on this issue soon.

Acknowledgements I am very grateful to B. Sathiapalan, N. Suryanarayana, R. Kaul and D. Dudal for useful discussions and for giving me valuable comments. I would like to thank A. Dey, Zodinmawia, A. Sharma and D. Dudal for careful reading of the manuscript and pointing out the necessary corrections. Part of this work was done while the author was in The Institute of Mahematical Sciences, Chennai and was funded by a Postdoctoral fellowship provided by the Department of Atomic Energy of the Government of India. This work is partially supported by the postdoctoral grant PDM/15/172 from KU Leuven.

Open Access This article is distributed under the terms of the Creative Commons Attribution 4.0 International License (http://creativecomm ons.org/licenses/by/4.0/), which permits unrestricted use, distribution, and reproduction in any medium, provided you give appropriate credit to the original author(s) and the source, provide a link to the Creative Commons license, and indicate if changes were made.

Funded by SCOAP ${ }^{3}$.

\section{References}

1. M.A. Nielsen, I.L. Chuang, Quantum computation and quantum information (Cambridge University Press, Cambridge, 2010)

2. P. Calabrese, J.L. Cardy, Entanglement entropy and quantum field theory. J. Stat. Mech. 0406, P002 (2004). arXiv:hep-th/0405152

3. J.M. Maldacena, The large $\mathrm{N}$ limit of superconformal field theories and supergravity. Adv. Theor. Math. Phys. 2, 231 (1998). arXiv:hep-th/9711200

4. S. Ryu, T. Takayanagi, Holographic derivation of entanglement entropy from AdS/CFT. Phys. Rev. Lett. 96, 181602 (2006). arXiv:hep-th/0603001
5. S. Ryu, T. Takayanagi, Aspects of holographic entanglement entropy. JHEP 08, 045 (2006). arXiv:hep-th/0605073

6. M. Headrick, General properties of holographic entanglement entropy. JHEP 03, 085 (2014). arXiv: 1312.6717

7. A. Lewkowycz, J. Maldacena, Generalized gravitational entropy. JHEP 08, 090 (2013). arXiv:1304.4926

8. H. Casini, M. Huerta, R.C. Myers, Towards a derivation of holographic entanglement entropy. JHEP 1105, 036 (2011). arXiv: 1102.0440

9. H. Casini, M. Huerta, Entanglement entropy for the n-sphere. Phys. Lett B 694, 167 (2010). arXiv:1007.1813

10. L.Y. Hung, R.C. Myers, M. Smolkin, A. Yale, Holographic calculations of Rényi entropy. JHEP 1112, 047 (2011). arXiv:1110.1084

11. X. Dong, The gravity dual of renyi entropy. Nat. Commun. 7, 12472 (2016). arXiv: 1601.06788

12. M. Headrick, Entanglement Rényi entropies in holographic theories. Phys. Rev. D 82, 126010 (2010). arXiv:1006.0047

13. T. Faulkner, The entanglement Rényi entropies of disjoint intervals in AdS/CFT. arXiv: 1303.7221

14. R.K. Kaul, P. Majumdar, Logarithmic correction to the Bekenstein-Hawking entropy. Phys. Rev. Lett. 84, 5255 (2000). arXiv:gr-qc/0002040

15. S. Banerjee, R.K. Gupta, A. Sen, Logarithmic corrections to extremal black hole entropy from quantum entropy function. JHEP 03, 147 (2011). arXiv: 1005.3044

16. S. Banerjee, R.K. Gupta, I. Mandal, A. Sen, Logarithmic corrections to $\mathrm{N}=4$ and $\mathrm{N}=8$ black hole entropy: a one loop test of quantum gravity. JHEP 1111, 143 (2011). arXiv: 1106.0080

17. A. Sen, Logarithmic corrections to Schwarzschild and other nonextremal black hole entropy in different dimensions. JHEP 1304, 156 (2013). arXiv:1205.0971

18. S. Bhattacharyya, A. Grassi, M. Marino, A. Sen, A one-loop test of quantum supergravity. Class. Quant. Grav. 31, 015012 (2014). arXiv: 1210.6057

19. S.N. Solodukhin, The Conical singularity and quantum corrections to entropy of black hole. Phys. Rev. D 51, 609 (1995). arXiv:hep-th/9407001

20. S.N. Solodukhin, On nongeometric contribution to the entropy of black hole due to quantum corrections. Phys. Rev. D 51, 618 (1995). arXiv:hep-th/9408068

21. D.V. Fursaev, Temperature and entropy of a quantum black hole and conformal anomaly. Phys. Rev. D 51, 5352 (1995). arXiv:hep-th/9412161

22. E. Shaghoulian, Black hole microstates in AdS. Phys. Rev. D 94(10), 104044 (2016). arXiv: 1512.06855

23. S. Das, P. Majumdar, R.K. Bhaduri, General logarithmic corrections to black hole entropy. Class. Quant. Grav. 19, 2355 (2002). arXiv:hep-th/0111001

24. G. Gour, A.J.M. Medved, Thermal fluctuations and black-hole entropy. Class. Quant. Grav. 20, 3307 (2003). arXiv:gr-qc/0305018

25. A. Chatterjee, P. Majumdar, Mass and charge fluctuations and black hole entropy. Phys. Rev. D 71, 024003 (2005). arXiv:gr-qc/0409097

26. S. Mukherji, S.S. Pal, Logarithmic corrections to black hole entropy and AdS/CFT correspondence. JHEP 05, 026 (2002). arXiv:hep-th/0205164

27. S. Mahapatra, P. Phukon, Tapobrata Sarkar, Black hole entropy corrections in the grand canonical ensemble. Phys. Rev. D 84, 044041 (2011). arXiv:1103.5885

28. J.D. Brown, M. Henneaux, Central charges in the canonical realization of asymptotic symmetries: an example from three dimensional gravity. Commun. Math. Phys. 104, 207 (1986)

29. A. Strominger, Black hole entropy from near horizon microstates, JHEP 02, 009 (1998). arXiv:hep-th/9712251

30. S. Carlip, What we dont know about BTZ black hole entropy, Class. Quant. Grav. 15, 3609 (1998). arXiv:hep-th/9806026 
31. S. Carlip, Black hole entropy from conformal field theory in any dimension. Phys. Rev. Lett. 82, 2828 (1999). arXiv:hep-th/9812013

32. S. Carlip, Entropy from conformal field theory at Killing horizons. Class. Quantum Grav. 16, 3327 (1999). arXiv:gr-qc/9906126

33. W. Donnelly, A. C. Wall, Geometric entropy and edge modes of the electromagnetic field, Phys. Rev. D 94(10), 104053 (2016). arXiv: 1506.05792

34. J.A. Cardy, Operator content of two dimensional conformally invariant theories. Nucl. Phys. B 270, 186 (1986)

35. S. Carlip, Logarithmic corrections to black hole entropy from the Cardy formula, Class. Quant. Grav. 17, 4175 (2000). arXiv:gr-qc/0005013

36. B.R. Majhi, T. Padmanabhan, Noether current from the surface term of gravitational action, Virasoro algebra, and horizon entropy. Phys. Rev. D 86, 101501(R) (2012). arXiv: 1204.1422

37. H. Liu, M. Mezei, A Refinement of entanglement entropy and the number of degrees of freedom. JHEP 1304, 162 (2013). arXiv: 1202.2070

38. C. Beck, F. Schlogl, Thermodynamics of chaotic systems (Cambridge University Press, Cambridge, 1993)

39. K. Zyczkowski, Rényi extrapolation of Shannon entropy. Open Syst. Inf. Dyn. 10, 297 (2003). arXiv:quant-ph/0305062

40. T. Faulkner, A. Lewkowycz, J. Maldacena, Quantum corrections to holographic entanglement entropy. JHEP 11, 074 (2013). arXiv:1307.2892

41. T. Barrella, X. Dong, S.A. Hartnoll, V.L. Martin, Holographic entanglement beyond classical gravity. JHEP 09, 109 (2013). arXiv: 1306.4682

42. E. Shaghoulian, Modular forms and a generalized Cardy formula in higher dimensions. Phys. Rev. D 93, 126005 (2016). arXiv: 1508.02728

43. F. Loran, M.M.S. Jabbarib, M. Vinconb, Beyond logarithmic corrections to Cardy formula. JHEP 01, 110 (2011). arXiv:1010.3561

44. B.R. Majhi, T. Padmanabhan, Noether current, horizon Virasoro algebra, and entropy. Phys. Rev. D 85, 084040 (2012). arXiv:1111.1809
45. V. Iyer, R.M. Wald, Some properties of Noether charge and a proposal for dynamical black hole entropy. Phys. Rev. D 50, 846 (1994). arXiv:gr-qc/9403028

46. R.G. Cai, Gauss-Bonnet black holes in AdS spaces. Phys. Rev. D 65, 084014 (2002). arXiv:hep-th/0109133

47. S.C. Davis, Generalized Israel junction conditions for a Gauss-Bonnet brane world. Phys. Rev. D 67, 024030 (2003). arXiv:hep-th/0208205

48. M. Cvetic, S. Nojiri, S.D. Odintsov, Black hole thermodynamics and negative entropy in de Sitter and anti-de Sitter Einstein-Gauss-Bonnet gravity. Nucl. Phys. B 628, 295 (2002). arXiv:hep-th/0112045

49. S. Nojiri, S.D. Odintsov, Anti-de Sitter black hole thermodynamics in higher derivative gravity and new confining deconfining phases in dual CFT. Phys. Lett. B 521, 87 (2001). arXiv:hep-th/0109122

50. S. Silva, Black hole entropy and thermodynamics from symmetries, Class Quant. Grav. 19, 3947 (2002). arXiv:hep-th/0204179

51. T. Nishioka, T. Takayanagi, AdS bubbles, entropy and closed string tachyons. JHEP 01, 090 (2007). arXiv:hep-th/0611035

52. E. Itou, K. Nagata, Y. Nakagawa, A. Nakamura, V. I. Zakharov, Entanglement in four-dimensional SU(3) gauge theory, Prog. Theor. Exp. Phys. 061B01 (2016). arXiv:1512.01334

53. D. Dudal, S. Mahapatra, Confining gauge theories and holographic entanglement entropy with a magnetic field. JHEP 04, 031 (2017). arXiv: 1612.06248

54. N. Engelhardt, A.C. Wall, Quantum extremal surfaces: holographic entanglement entropy beyond the classical regime. JHEP 01, 073 (2015). arXiv:1408.3203

55. M. Marino, P. Putrov, Interacting fermions and $\mathrm{N}=2$ ChernSimons-matter theories. JHEP 11, 199 (2013). arXiv:1206.6346

56. I.R. Klebanov, S.S. Pufu, S. Sachdev, B.R. Safdi, Rényi entropies for free field theories. JHEP 04, 074 (2012). arXiv:1111.6290

57. J .S. Dowker, Entanglement entropy for odd spheres. arXiv: 1012.1548 